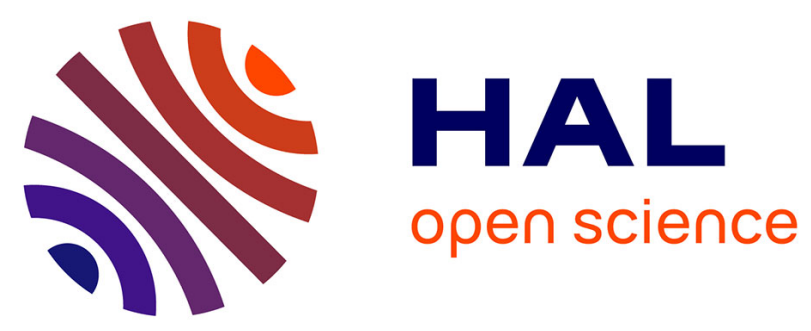

\title{
Resting-state posterior alpha rhythms are abnormal in subjective memory complaint seniors with preclinical Alzheimer's neuropathology and high education level: the INSIGHT-preAD study
}

Claudio Babiloni, Susanna Lopez, Claudio del Percio, Giuseppe Noce, Maria Teresa Pascarelli, Roberta Lizio, Stefan Teipel, Gabriel González-Escamilla, Hovagim Bakardjian, Nathalie George, et al.

\section{To cite this version:}

Claudio Babiloni, Susanna Lopez, Claudio del Percio, Giuseppe Noce, Maria Teresa Pascarelli, et al.. Resting-state posterior alpha rhythms are abnormal in subjective memory complaint seniors with preclinical Alzheimer's neuropathology and high education level: the INSIGHT-preAD study. Neurobiology of Aging, 2020, 90, pp.43-59. 10.1016/j.neurobiolaging.2020.01.012 . hal-03006214

HAL Id: hal-03006214

https://hal.science/hal-03006214

Submitted on 5 Jan 2021

HAL is a multi-disciplinary open access archive for the deposit and dissemination of scientific research documents, whether they are published or not. The documents may come from teaching and research institutions in France or abroad, or from public or private research centers.
L'archive ouverte pluridisciplinaire HAL, est destinée au dépôt et à la diffusion de documents scientifiques de niveau recherche, publiés ou non, émanant des établissements d'enseignement et de recherche français ou étrangers, des laboratoires publics ou privés. 


\title{
Resting-state posterior alpha rhythms are abnormal in subjective memory complaint seniors with preclinical Alzheimer's neuropathology and high education level: the INSIGHT-preAD study
}

\author{
Claudio Babiloni ${ }^{\mathrm{a}, \mathrm{b}, *, 1}$, Susanna Lopez ${ }^{\mathrm{a}, \mathrm{c}, 1}$, Claudio Del Percio ${ }^{\mathrm{a}}$, Giuseppe Noce ${ }^{\mathrm{d}}$, \\ Maria Teresa Pascarelli ${ }^{\mathrm{e}}$, Roberta Lizio ${ }^{\mathrm{d}}$, Stefan J. Teipel ${ }^{\mathrm{f}, \mathrm{g}}$, Gabriel González-Escamilla ${ }^{\mathrm{h}}$,
} Hovagim Bakardjian $^{\mathrm{i}, \mathrm{j}}$, Nathalie George ${ }^{\mathrm{k}}$, Enrica Cavedo ${ }^{\mathrm{i}, \mathrm{j}, 1}$, Simone Lista ${ }^{\mathrm{i}, \mathrm{j}, \mathrm{l}}$, Patrizia Andrea Chiesa ${ }^{\mathrm{i}, \mathrm{j}, 1}$, Andrea Vergallo, Pablo Lemercier ${ }^{\mathrm{i}, \mathrm{j}, 1}$, Giuseppe Spinelli ${ }^{\mathrm{i}, \mathrm{j}}$, Michel J. Grothe ${ }^{\mathrm{g}}$, Marie-Claude Potier ${ }^{\mathrm{j}}$, Fabrizio Stocchi ${ }^{\mathrm{m}}$, Raffaele Ferri ${ }^{\mathrm{e}}$, Marie-Odile Habert $^{\mathrm{n}, \mathrm{o}, \mathrm{p}}$, Francisco J. Fraga ${ }^{\mathrm{q}}$, Bruno Dubois ${ }^{\mathrm{i}, \mathrm{j}}$, Harald Hampel ${ }^{\mathrm{l}}$, INSIGHT-preAD Study Group

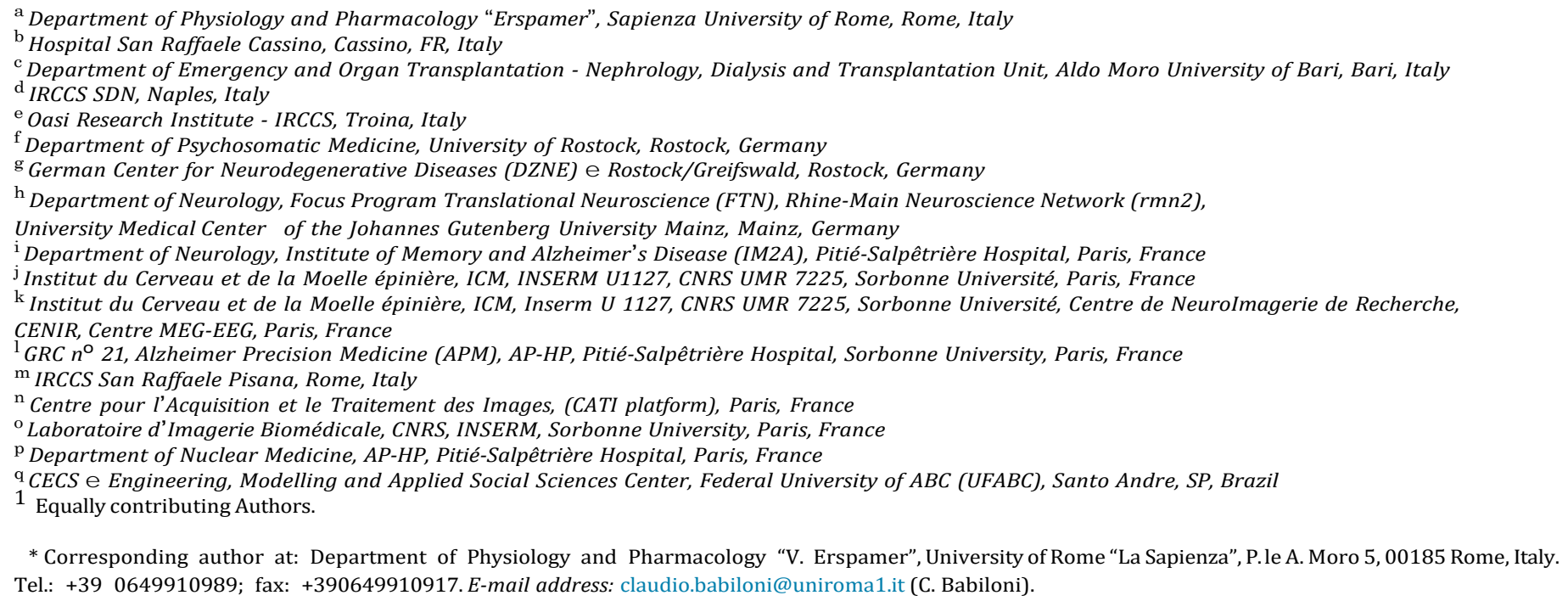

\section{A B STRACT}

Cognitive reserve is present in Alzheimer's disease (AD) seniors with high education attainment making them clinically resilient to extended brain neuropathology and neurodegeneration. Here, we tested whether subjective memory complaint (SMC) seniors with $\mathrm{AD}$ neuropathology and high education attainment of the prospective INSIGHT-preAD cohort (Paris) may present abnormal eyesclosed resting state posterior electroencephalographic rhythms around individual alpha frequency peak, typically altered in $\mathrm{AD}$ patients. The SMC participants negative to amyloid PET AD markers (SMCneg) with high (over low-moderate) education level showed higher posterior alpha 2 power density (possibly "neuro-protective"). Furthermore, amyloid PET-positive SMC (SMCpos) participants with high (over low-moderate) education level showed higher temporal alpha 3 power density (possibly "neuro-protective") and lower posterior alpha 2 power density (possibly "compensatory"). This effect may reflect cognitive reserve as no differences in brain graywhite matter, and cognitive functions were observed between these SMCpos/SMCneg subgroups. Preclinical Alzheimer's neuropathology may interact with education attainment and neurophysiological mechanisms generating cortical alpha rhythms around individual alpha frequency peak (i.e., alpha 2 and 3) in quiet wakefulness.

\section{Keywords:}

Resting state EEG rhythms Alpha rhythms

Preclinical Alzheimer's disease (AD) Preclinical Alzheimer's neuropathology Subjective memory complaint (SMC) INSIGHTpreAD study 
According to the international guidelines for the in vivo diagnosis of Alzheimer's neuropathology in clinical research (Albert et al., 2011; Dubois et al., 2014; Jack et al., 2018; McKhann et al., 2011), abnormal deposition of amyloid- $B$ in the brain can be revealed by Ab1-42 measured in the cerebrospinal fluid (CSF) or standard uptake value ratio (SUVR) of amyloid-sensitive positron emission tomography (PET) tracers, such as ${ }^{18} \mathrm{~F}$-florbetapir, florbetaben, flutemetamol, or ${ }^{11} \mathrm{C}-\mathrm{PIB}$. Furthermore, Alzheimer's disease (AD) neuropathology can be revealed by both AB42 and phosphor-tau in the CSF and tau PET load (Albert et al., 2011; Dubois et al., 2014; Jack et al., 2018; McKhann et al., 2011). The downstream effects of AD neuropathology on structural (i.e., neurodegeneration) and functional brain integrity can be evaluated by (1) structural magnetic resonance imaging (sMRI) of hippocampus and cortical gray matter and (2) ${ }^{18}$ F-Fluorodeoxyglucose-positron emission tomography (FDG-PET) of metabolic activity (i.e., quiet wakefulness) in hippocampus and cerebral cortex during the resting state condition (Albert et al., 2011; Dubois et al., 2014; Jack et al., 2018; McKhann et al., 2011).

Magnetic resonance imaging (MRI) and FDG-PET markers have shown that in older adults, high education attainment, indexes of intellectual activity in life span, verbal intelligent quotient, and residual variance in episodic memory scores after accounting for brain and demographic variables may represent the so-called "cognitive reserve" (CR), which may be associated with both protective and compensatory mechanisms in relation to pathological brain aging (Devanand et al., 2013; Reed et al., 2010; Stern et al., 2018; Zahodne et al., 2013, 2015). Neuroprotection mechanisms of CR may maintain a relative structural integrity of brain over age ("brain-maintenance"), whereas compensatory mechanisms may make older adults clinically resilient to extended brain neuropathologies and neurodegeneration (Barulli and Stern, 2013; Nyberg et al., 2012; Stern et al., 2018). Specifically, compensatory mechanisms of CR may attenuate or annul (i.e., "moderate") the expected linear relationship between structural-functional integrity of the brain and cognitive status in older people. When CR is depleted, patients' cognitive status may quickly worsen in older adults not only with AD neuropathology (Amieva et al., 2014; Bennett et al., 2003; Chaves et al., 2010; Hall et al., 2007; Helzner et al., 2009; Meng and D'Arcy, 2013; Mungas et al., 2018; Roe et al., 2007; Scarmeas et al., 2006; Stern, 2012; Stern et al., 1999, 2018; Weiner et al., 1998; Wilson et al., 2000, 2004, 2019) but also with blood systolic hypertension and brain white matter hyperintensity (Zahodne et al., 2019).

The above findings encourage longitudinal observational studies lasting several years, including serial measurements of brain and cognitive functions in seniors with intact cognitive status and AD patients having various education levels. However, the repeated recordings of neuroimaging data may be challenging because of their invasiveness and high costs. Therefore, lower-threshold alternative techniques for brain research are under evaluation. An interesting low-cost, noninvasive, repeatable over time, and worldwide available candidate technique is the recording and quantitative spectral analysis of scalp electroencephalographic (EEG) rhythms in resting individuals with eyes closed instructed to maintain quiet wakefulness and vigilance for a while, the so-called resting state condition. In this condition, seniors are asked to remain comfortably seated and relaxed in a silent room, without substantial goal-oriented mentalizations. The EEG recording typically shows resting state posterior electroencephalographic (rsEEG) oscillatory activity characterized by ample alpha rhythms at $8 \mathrm{e} 12 \mathrm{~Hz}$ frequencies distributed in posterior regions of the scalp (PfurtschellerandLopes daSilva,1999). Theseamplealpharhythms may reflect oscillatory neurophysiological mechanisms of thalamuscortical neural synchronization related to the thalamic gating of neural signals arousing cerebral cortex with a general effect of cortical inhibition of primary and associative cortical areas (Babiloni et al., 2016a; Pfurtscheller and Lopes da Silva, 1999).

In physiological aging, alpha rhythms exhibit a progressive reduction in amplitude in relation to the deterioration in fiber tracts linking cholinergic basal forebrain, thalamus, and visual cortex (Babiloni et al., 2006a, 2009; Wan et al., 2019). This relation has been also corroborated by in vitro studies of thalamocortical alpha rhythms in mice (Hughes and Crunelli, 2005; Lörincz et al., 2008, 2009).

In pathological aging, previous rsEEG investigations have used low-resistance EEG recordings from 10 to 20 montage system (i.e., 19 scalp electrodes) to demonstrate abnormalities in alpha and other rhythms at different AD stages. Compared with seniors with intact cognition, AD patients with dementia (ADD) were characterized by lower posterior alpha (8-12 Hz) and beta (13-30 Hz) rhythms (Babiloni et al., 2006a,b,c; Dierks et al., 1993, 2000; Jelic et al., 2000; Jeong, 2004; Ponomareva et al., 2003). Furthermore, ADD exhibited higher delta $(<4 \mathrm{~Hz})$ and theta $(4-7 \mathrm{~Hz})$ rhythms widely distributed over the scalp (Brassen and Adler, 2003; Kogan et al., 2001; Onofrj et al., 2003; Reeves et al., 2002; Rodriguez et al., 2002; Valladares-Neto et al., 1995). These abnormalities in rsEEG rhythms were related to genetic disease risk factors, cholinergic therapy, vascular lesions of brain white matter as well as regional cerebral blood flow and atrophy of posterior cortical gray matter and hippocampus (Babiloni et al., 2006a,b,c, 2013, 2015, 2016; Buchan et al., 1997; Kudoh et al., 1997; Rodriguez et al., 1998, 1999, 2004; Szelies et al., 1999).

Concerning prodromal AD stages, previous rsEEG investigations also showed that AD patients with mild cognitive impairment (ADMCI) were characterized by abnormally low amplitude of posterior alpha rhythms and higher amplitude of delta-theta rhythms, in association with the mentioned abnormalities in brain structure and alterations in functional connectivity within cortical default mode network (Babiloni et al., 2006a, 2013, 2015, 2018; Galluzzi et al., 2016; Jovicich et al., 2019; Marizzoni et al., 2019). These changes in rsEEG rhythms exhibited some peculiar features when compared with those observed in patients suffering from MCI to dementia because of Parkinson's and Lewy Body diseases (Babiloni et al., 2017, 2018).

In an earlier AD stage, namely that of subjective memory complaint (SMC), previous rsEEG investigations showed mixed results. On one hand, it was reported that SMC participants (amyloid deposition in the brain not tested) were characterized by elevated amplitude of alpha rhythms negatively correlated with language and memory scores and positively correlated with reversed digit span scores (Alexander et al., 2006). In the same line, SMC patients with amyloid deposition in the brain (i.e., preclinical Alzheimer's neuropathology) presented an increase in amplitude of alpha rhythms estimated in medial frontal cortex by magnetoencephalographic (MEG) techniques (Nakamura et al., 2018). On the other hand, other EEG and MEG evidence showed decreased amplitude of posterior alpha rhythms in quiet vigilance. Compared with a control group of older adults without SMC and cognitive deficits, both SMC and MCI seniors were characterized by lower amplitude of posterioralpharhythmsin rsEEGand rsMEGrhythms in relation to cognitive functions (Babiloni et al., 2010; López-Sanz et al., 2016). Furthermore, SMC participants with amyloid deposition in the brain (i.e., preclinical Alzheimer's neuropathology) exhibited higher amplitude of delta-theta rhythms and lower amplitude and frequency of alpha rhythms in relation to clinical worsening over time (Gouw et al., 2017).

The monocentric observational INSIGHT-preAD study reported other relevant evidence. In that study, SMC seniors were enrolled to investigate optimal biomarkers of AD progression from preclinical stages (Dubois et al., 2018), including rsEEG rhythms at alpha and other frequencies (i.e., EEG recordings by 256-Channel Geodesic EEG System, Electrical Geodesics, Inc, EGI). The diagnosis of preclinical Alzheimer's neuropathology in SMC (SMCpos) seniors was based on the positivity to SUVR of 18F-florbetapir PET (Dubois et al., 2018). Up to now, two articles reported the first rsEEG findings of INSIGHT-preAD study. They are summarized in the following. In the first study (Teipel et al., 2017a), results showed only a statistical trend in the relationship between global 18F-florbetapir PET SUVR and posterior alpha 
rhythms ( $p<0.05$ not corrected for multiple statistical comparisons). In the second study (Gaubert et al., 2019), results showed a nonlinear U-shaped relationship of $18 \mathrm{~F}$-florbetapir PET SUVR with beta and gamma rhythms, whereas the relationship with alpha rhythms remained unclear.

Taken together, the findings of these two INSIGHT-preAD articles suggest that the preclinical Alzheimer's neuropathology may affect rsEEG rhythms in SMC seniors but with unclear consequences on posterior alpha rhythms. In both articles, effects of the participants' education level were not considered. In the present retrospective study, we re-analyzed the rsEEG data of the INSIGHT-preAD cohort with the aim to test the hypothesis that SMC seniors with in vivo evidence of Alzheimer's neuropathology, and high education level may show first signs of abnormalities in resting-state posterior alpha rhythms as a neurophysiological counterpart of the picture emerging from the sMRI and FDG-PET evidence reported above. To ensure the spatial-frequency comparison of the present results with those of our previous rsEEG studies mentioned above (Babiloni et al., 2010, 2017, 2018; Galluzzi et al., 2016; Jovicich et al., 2019), we considered only electrodes of the traditional 10-20 montage system (i.e., 19 scalp electrodes), carefully controlled as quality of EEG signals and lack of biological and instrumental artifacts (see Methods). Furthermore, the primary analysis of the study took into account the individual variation of alpha frequency peak in rsEEG rhythms in line with recent reference studies of rsEEG rhythms in older control adults and AD patients (Babiloni et al., 2017, 2018; Gouw et al., 2017).

\section{Materials and methods}

\subsection{Participants}

Participants were recruited at the Pitié-Salpêtrière University Hospital in Paris, France, in the framework of the INSIGHT-preAD study with the objective to investigate the earliest preclinical stages of $\mathrm{AD}$ and its development including influencing factors and markers of disease progression (Dubois et al., 2018). The INSIGHT-preAD study currently includes baseline data of 318 cognitively normal individuals, between 70 and 85 year old, with SMCs and unimpaired cognition (i.e., Mini Mental State Examination [MMSE] score ?_27 and Clinical Dementia Rating score equal to 0) and with defined brain amyloid status. Demographic, cognitive, functional, nutritional, biological, genetic, genomic, imaging, electrophysiological, and other assessments were performed at baseline (see Dubois et al., 2018 for details). No evidence of episodic memory deficit was observed among participants, with a total recall at the Free and Cued Selective Reminding Test in the normal range (mean $46.1 \pm 2.0$; Teipel et al., 2017a,b). SMCs were confirmed by an affirmative answer to both of the following questions: (1) "Are you complaining about your memory?" and (2) "Is it a regular complaint which lasts more than 6 months?".

All experiments were performed with the informed and overt consent of each participant or caregiver, in line with the Code of Ethics of the World Medical Association (Declaration of Helsinki) and the standards established by the local Institutional Review Board. Local institutional Ethics Committee approved data sharing for scientific purpose.

\subsection{MRI acquisition}

MRI images were acquired using a 3 Tesla scanner with parallel imaging capabilities (Siemens Magnetom Verio, Siemens Medical Solutions, Erlangen, Germany). A quadrature detection head coil with 12 channels (transmit-receive circularly polarized CP-head coil) was used. For the anatomical structural study, 3D Turbo-FLASH sequences were performed (orientation sagittal; repetition time 2300 ms; echo time $2.98 \mathrm{~ms}$; inversion time $900 \mathrm{~ms}$; flip angle $9^{\circ}$; 176 slices; slice thickness $1 \mathrm{~mm}$; field of view $256 * 240 \mathrm{~mm}$; matrix $256 * 240$; bandwidth $240 \mathrm{~Hz} / \mathrm{Px}$; see Teipel et al., 2017a,b for more details).

In this study, we used biomarkers of sMRIs derived in the INSIGHT-preAD cohort in a previous investigation of this Workgroup focused on those biomarkers (Cavedo et al., 2018; Teipel et al., 2017a,b). Full details of the standard procedures for the computation of the biomarkers reported in the present tables and wording can be found in the original article reporting the results of that investigation (Teipel et al., 2017a,b).

\subsection{PET data acquisition and processing}

PET scans were acquired 50 minutes after injection of $370 \mathrm{MBq}(10 \mathrm{mCi}){ }^{18} \mathrm{~F}$-florbetapir. Reconstructed PET images were analyzed with a pipeline developed by the CATI (http://cati-neuroimaging. com/). sMRI images were co-registered to ${ }^{18}$ Florbetapir-PET images using SPM8 with visual inspection to detect any coregistration errors. Inverse deformation fields and matrix transformation from MRI data processing were used to derive composite cortical regions of interest (ROIs) (left and right precuneus, posterior and anterior cingulate, parietal, temporal, and orbitofrontal cortex; according to Clark et al., 2012), and a reference region (in pons and whole cerebellum) was placed in the individual native PET space. RBV-sGTM method was used to correct for partial volume effect (Thomas et al., 2011). Parametric PET images were created for each individual, by dividing each voxel with the mean activity extracted from the reference region. Finally, SUVR values were calculated by averaging the mean activity of all cortical ROIs in the individual PET native space.

To stratify participants into two groups of amyPET-positive (SMCpos) and amyPET-negative (SMCneg), we used the specific SUVR thresholds identified in a previous investigation of this Workgroup focused on those biomarkers (Habert et al., 2018). Full details of the procedures for the computation of those thresholds can be found in the original article reporting the results of that investigation (Habert et al., 2018). Summarizing, those procedures identified two thresholds, one more conservative (0.88) and the other more liberal (0.79). The more conservative threshold was used in the previous reference study investigating functional brain connectivity from rsEEG alpha and beta rhythms in the INSIGHT-preAD cohort (Teipel et al., 2017a,b). The liberal threshold has been adopted for several parallel rsEEG and neuroimaging studies in the INSIGHT-preAD cohort (Dubois et al., 2018). Therefore, for the present study, we opted for the value of 0.79 for the main rsEEG data analysis. The statistically significant results of such rsEEG data analysis $(p<0.05$ corrected) were, then, cross-validated with that based on the value of 0.88 . 


\subsection{EEG recordings}

EEG data were recorded while the participants were sitting comfortably and relaxed with eyes closed in a standard resting state condition. At least 120 seconds of rsEEG (e.g., 2 periods of eyes-closed condition lasting 30 seconds each, intermingled with 2 periods of eyes-open condition) were acquired using a high-density 256-channel EGI system (Electrical Geodesics Inc, Eugene, OR, USA) with a sampling rate of $250 \mathrm{~Hz}$ and antialiasing band-pass analogic filtering. In this EGI system, the electrodes used are sponge-based to have a quick application time (10-20 minutes), ideal for seniors. Among the 256 electrodes, 224 electrodes cover the whole scalp while the remaining ones are placed on the front, top neck, and the face, allowing the measurement of electro-oculographic and muscular electromyographic (EMG) activity. The impedances of all scalp electrodes were kept below 50 KOhm. The reference electrode was placed at $\mathrm{Cz}$ site. A cephalic ground was used.

\subsection{Spectral and quality analysis of the rSEEG data}

An important step of the present methodological approach was to minimize the inter-individual variance of rsEEG rhythms with a procedure able to minimize (1) confounding effects of border electrodes with high impedance; (2) rsEEG data with residual artifacts provoked by head or eye muscle activities; and (3) individual variability in the alpha frequency power peak (i.e., using individual alpha frequency peak, IAFp, as a benchmark; Gonzalez-Escamilla et al., 2015, 2016; Klimesch, 1999). This goal was reached by a semiautomatic "pruning" procedure of preliminary rsEEG data analysis. Specifically, the "pruning" procedure was designed (Dr Susanna Lopez and Prof. Claudio Babiloni) and applied by two experts (Dr Susanna Lopez and Dr Claudio Del Percio) of the Group of Sapienza University of Rome to discard individual rsEEG segments, single electrodes, or the whole rsEEG data sets with long periods ( $>20$ seconds) of irremediable artifacts. To ensure rsEEG recordings of sufficient quality and an optimal comparison with previous rsEEG findings obtained in international projects (e.g., European FP7 IMI project with the short name "PharmaCog" performed in AD patients with amnesic MCI [ADMCI; www.pharmacog.org/]), we considered only 19 scalp electrodes of the traditional 10-20 montage system (i.e., Fp1, Fp2, F7, F3, Fz, $\mathrm{F} 4, \mathrm{~F} 8, \mathrm{~T} 3, \mathrm{C} 3, \mathrm{Cz}, \mathrm{C} 4, \mathrm{~T} 4, \mathrm{~T} 5, \mathrm{P} 3, \mathrm{Pz}, \mathrm{P} 4, \mathrm{~T} 6, \mathrm{O} 1$, and $\mathrm{O} 2$ ) from the whole array available.

According to the mentioned "pruning" procedure, an individual rsEEG data set of the present INSIGHT-preAD database was defined as "artifact freeewith sufficient quality" and usable for the final rsEEG data analysis if it passed the following control steps:

(1) Mathematical removal (detrending) of the slow fluctuation from the recorded scalp rsEEG traces (10-20 electrode montage system) was performed using the popular EEGLAB toolbox (https://sccn.ucsd.edu/eeglab/index.php). Afterward, the rsEEG traces (10-20 electrode montage system) were expected to cross the isoelectric lines.

(2) The first visual analysis of the detrended rsEEG traces (10-20 electrode montage system) was performed to discard the rsEEG segments showing instrumental (bad electric contact electrode-scalp) artifacts that were considered as "irremediable" by the two experts working independently. More than 40 seconds of EEG traces without those "irremediable artifacts" had to be available for the following step;

(3) EEGLAB independent component analysis and removal of the estimated independent components showing residual instrumental or biological (i.e., electrooculographic and EMG) artifacts were performed. EEGLAB was used to reconstruct rsEEG signals without those artifactual components. The second visual analysis of the EEG traces (10-20 electrode montage system) served to control the quality of the detrended and reconstructed rsEEG traces without noise-related independent components. This analysis aimed at discarding rsEEG segments showing residual instrumental or biological artifacts. The individual rsEEG data sets were accepted for the following step only if they were formed by > 40 seconds of artifact-free EEG activity;

(4) For each subject, rsEEG data were re-referenced to the common average among all artifact-free electrodes of 10-20 montage system. This procedure regenerated the voltage values at $\mathrm{Cz}$ used as physical reference electrode during the rsEEG recordings. Afterward, we used a standard digital fast Fourier transform for the computation of power density spectra from the artifact-free rsEEG traces (Welch technique, Hanning windowing function, no phase shift). The power density spectra were computed from the artifact-free 2 -s rsEEG epochs with

$0.5 \mathrm{~Hz}$ of frequency resolution and averaged across all these epochs. Afterward, these rsEEG power density spectra were normalized with the following procedure: the absolute rsEEG power-density mean was calculated averaging the power density values for each electrode (10-20 electrode montage system) and frequency bin from 0.5 to $45 \mathrm{~Hz}$. Afterward, any absolute rsEEG power density value at a given electrode and frequency bin was divided by the absolute rsEEG power-density mean to obtain the normalized rsEEG power density values. In these values, one was equal to the absolute rsEEG power density mean across all electrodes and frequency bins. The operation was repeated for all frequency bins from 0.5 to $45 \mathrm{~Hz}$. All normalized rsEEG power density spectra of this step were used for the following step.

(5) The third visual analysis of the averaged (normalized) rsEEG power density spectra (10-20 electrode montage system) was performed to discard the individual rsEEG data sets associated with artifactual shapes of those spectra. Artifactual shapes were defined as characterized by the following features:

- Lack of the physiological decrease of the EEG power density after the IAFp as a function of the increase of the frequencies in the range of $1-40 \mathrm{~Hz}$

- Presence of an offset of power density across all frequencies at some scalp electrodes (especially visible as big differences in gamma rsEEG power density among the ROIs);

- Presence of many peaks of high-power density in the range of 1-40 Hz;

- Lack of a visible IAFp in the range between $6 \mathrm{~Hz}$ and $14 \mathrm{~Hz}$. When an rsEEG experiment in participants with normal brain structure and cognitive status is of sufficient quality, a visible IAFp is expected in rsEEG power density spectra at posterior scalp electrodes. To minimize the probability that the lack of IAFp be because of preclinical AD neuropathology rather than substantial artifacts, we considered only seniors with raw MMSE scores equal or greater an arbitrary value of 28 . The rationale was that even an SMCpos individual with preclinical Alzheimer's neuropathology (but without remarkable abnormalities in sMRIs based as revealed by visual inspection of the medical and radiological staff involved in the formulation of the diagnosis of the present SMC participants) should show a visible IAFp in association with MMSE scores ?_28 and neuropsychological test scores in the normal range. 
After this "pruning" procedure, 118 SMCneg and 54 SMCpos seniors with MMSE scores ?_28 and accepted rsEEG data sets were identified for the final data analysis. Table 1 summarizes the relevant demographic, neuropsychological (MMSE score), and genetic (Apolipoprotein $\varepsilon$ genotype,APOE) markers of these participants. In the table, we also report the results of the statistical analyses $(p<0.05)$ computed to evaluate the presence or absence of statistically significant differences between the two SMC groups for the age, sex, education, MMSE score, and APOE genotype. As expected, a statistically significant difference was found between the two SMC groups for the APOE genotype $\left(\chi^{2}=12.3 ; p<0.05\right)$. Specifically, there was a higher number of participants with APOE $\varepsilon 4$ genotype in the SMCpos than the SMCneg group $(p<0.05)$. On the contrary, no statistically significant difference was found for the age, sex, education, and MMSE score between the SMCpos and SMCneg groups $(p>0.05)$.

The rsEEG frequency bands of interest were individually identified based on the following frequency landmarks: the transition frequency (TF) and the IAFp (Klimesch, 1999; Klimesch et al., 1996). The TF marks the transition frequency between the theta and alpha bands defined as the minimum of the rsEEG power density between $3 \mathrm{~Hz}$ and $8 \mathrm{~Hz}$ (i.e., between the delta and the alpha power peak), whereas the IAFp is defined as the maximum power density peak between $6 \mathrm{~Hz}$ and $14 \mathrm{~Hz}$. The reference values to compute TF and IAFp on individual basis were obtained averaging rsEEG power density across $\mathrm{Fz}, \mathrm{Cz}, \mathrm{Pz}, \mathrm{O} 1$, and $\mathrm{O} 2$ electrodes, which ensure the recording of scalp EEG signals with low biological noise (e.g., the scalp midline is relatively far from temporal and frontal muscles) and optimal electrical contacts using a standard EEG helmet. Furthermore, those selected electrodes were especially suitable to record rsEEG rhythms at delta/theta (Fz and $\mathrm{Cz})$ and alpha (Pz, O1, and $\mathrm{O} 2$ ) frequencies in our previous reference field studies $(2017,2018)$.

In detail, the following individual frequency bands were used in the present study considering a frequency resolution of $0.5 \mathrm{~Hz}$ :

(1) delta from $\mathrm{TF}-4 \mathrm{~Hz}$ to $\mathrm{TF}-2 \mathrm{~Hz}$;

(2) theta from $\mathrm{TF}-2 \mathrm{~Hz}$ to $\mathrm{TF}$;

(3) alpha 1 from TF to the midpoint of the TF-IAFp range;

(4) alpha 2 from the midpoint of the TF-IAFp range to IAFp;

(5) alpha 3 from IAFp to IAFp $+2 \mathrm{~Hz}$.

Table 1

Mean values ( \pm standard error mean, SE) of the demographic, neuropsychological, and genetic data, together with the results of their statistical comparisons $(p<0.05)$ in the groups of seniors with subjective memory complaint (SMC) found to be amyloid negative (SMCneg) and positive (SMCpos) to the marker of Alzheimer's neuropathology derived from ${ }^{18} \mathrm{~F}$-florbetapir positron emission tomography (amyPET)

\begin{tabular}{|c|c|c|c|}
\hline & \multicolumn{3}{|c|}{$\begin{array}{l}\text { Mean values ( } \pm \text { SE) of demographic, clinical, and } \\
\text { genetic data }\end{array}$} \\
\hline & SMCneg & SMCpos & Statistical analysis \\
\hline $\mathrm{N}$ & 118 & 54 & - \\
\hline Age & $75.7( \pm 0.3 \mathrm{SE})$ & $76.6( \pm 0.4 \mathrm{SE})$ & $t$ test: n.s. \\
\hline $\operatorname{Sex}(M / F)$ & $43 / 75$ & $20 / 34$ & Fisher test: n.s. \\
\hline Education & $6.2( \pm 0.2 \mathrm{SE})$ & $5.8( \pm 0.3 \mathrm{SE})$ & $t$ test: n.s. \\
\hline MMSE & $28.9( \pm 0.1 \mathrm{SE})$ & $28.7( \pm 0.1 \mathrm{SE})$ & $\begin{array}{l}\text { Mann-Whitney } \\
U \text { test: n.s. }\end{array}$ \\
\hline $\begin{array}{l}\text { APOE } \\
\text { genotype } \\
(\mathrm{N})\end{array}$ & $\begin{array}{l}\varepsilon 2 / \varepsilon 2(1), \varepsilon 2 / \varepsilon 3(17), \\
\varepsilon 3 / \varepsilon 3(84), \varepsilon 2 / \varepsilon 4(1), \\
\varepsilon 3 / \varepsilon 4(15), \varepsilon 4 / \varepsilon 4(0)\end{array}$ & $\begin{array}{l}\varepsilon 2 / \varepsilon 2(0), \varepsilon 2 / \varepsilon 3(4), \\
\varepsilon 3 / \varepsilon 3(33), \varepsilon 2 / \varepsilon 4(0), \\
\varepsilon 3 / \varepsilon 4(15), \varepsilon 4 / \varepsilon 4(2)\end{array}$ & $\begin{array}{l}\chi^{2} \text { test }(2 \times 6): \\
p<0.05\end{array}$ \\
\hline
\end{tabular}

Key: MMSE, Mini Mental State Examination; M/F, males/females; APOE, apolipo-

protein E; n.s., not significant $(p>0.05)$.

To clarify the procedure, please consider an example of $\mathrm{TF}=4.5 \mathrm{~Hz}$ and IAFp $=10 \mathrm{~Hz}$. The midpoint of the TF-IAF range would be rounded to the nearest $0.5 \mathrm{~Hz}$ frequency $(=7.25 \mathrm{~Hz}$, rounded to $7 \mathrm{~Hz}$ for alpha 1 and $7.5 \mathrm{~Hz}$ for alpha 2). As a result, the alpha 1 power density would correspond to the mean obtained averaging all frequency bins included between TF and the midpoint of the TF-IAF range, namely $4.5,5,5.5,6,6.5$, and $7 \mathrm{~Hz}$ frequency bins. In the same vein, the alpha 2 power density would correspond to the mean obtained averaging all the frequency bins included between the midpoint of the TF-IAF range and the IAFp, namely 7.5, 8, 8.5, 9, 9.5, and 10 $\mathrm{Hz}$ frequency bins.

In the present study, the mean TF was $=5.4 \mathrm{~Hz}( \pm 0.1 \mathrm{SE})$ in both SMCneg and SMCpos groups. The mean IAF was $9.3 \mathrm{~Hz}( \pm 0.1 \mathrm{SE})$ in the SMCneg group and $9.4 \mathrm{~Hz}( \pm 0.1 \mathrm{SE})$ in the SMCpos group, with the maximum value of IAFp $=11 \mathrm{~Hz}$. No statistically significant

differences between the two groups were observed for both TF and IAFp $(t$ test, $p>0.05)$.

Fixed beta $1(13-20 \mathrm{~Hz})$, beta $2(20-30 \mathrm{~Hz})$, and gamma $(30-40 \mathrm{~Hz})$ bands were also considered.

Five ROIs were used considering the frontal, central, parietal, occipital, and temporal electrodes of the traditional 10-20 electrode montage system. Specifically, these ROIs included, respectively, (1) F3, Fz, and F4 electrodes for the frontal region; (2) C3, Cz, and C4 electrodes for the central region; (3) P3, Pz, and P4 electrodes for the parietal region; and (4) O1 and O2 electrodes for the occipital region; and (5) T3, T4, T5, and T6 for the temporal region (Babiloni et al., 2006).

\subsection{Stratification of the SMC seniors based on the education level}

To test the effect of the education level on the present spectral rsEEG markers, we used the education score adopted in the INSIGHT-preAD project (Dubois et al., 2018). In this score, the level of education ranged from 1 to 8 , one being associated with the attendance of the only infant school and eight with the attendance of the highest education level in the population (i.e., bachelor, master's degree, or doctorate). All the present SMC participants were stratified in 2 subgroups based on this score. The Edu- participants ranged from 2 to 6 of the education score, which grouped the SMC seniors with low-moderate education level. Furthermore, the SMC Edu+ participants were characterized by seven or eight of the education score (Dubois et al., 2018), which grouped the SMC seniors with high education level.

Table 2 reports the relevant demographic, neuropsychological (MMSE score), and genetic (APOE genotype) markers in the SMCneg Edu-, SMCneg Edu+, SMCpos Edu-, and SMCpos Edu+ subgroups. It also reports the results of the statistical analyses $(p<0.05)$ computed 
to evaluate the presence or absence of statistically significant differences between the Edu- and Edu+ subgroups for both SMCneg and SMCpos seniors as age ( $t$ test), sex (Fisher test), education ( $t$-test), MMSE score (Mann-Whitney $U$ test), and APOE genotype $\left(\mathrm{X}^{2}\right.$ test). As expected, and based on the stratification criterion, a statistically significant difference in education was unsurprisingly found between the Edu- and Edu+ subgroups for both SMCneg and SMCpos seniors considered separately $(p<0.00001)$. On the contrary, no statistically significant difference was found for the age, sex, MMSE score, and APOE genotype between the Edu- and Edu+ subgroups for both SMCneg and SMCpos seniors considered separately $(p>0.05)$.

Table 2

Mean values ( \pm standard error mean, SE) of the demographic, neuropsychological, and genetic data, together with the results of their statistical comparisons $(p<0.05)$ in the groups of SMCpos and SMCneg seniors stratified according to the high (Edu+) or low (Edu-) educational level

\begin{tabular}{|c|c|c|c|c|c|}
\hline \multicolumn{6}{|c|}{ Mean values ( \pm SE) of demographic, clinical, and genetic data } \\
\hline & SMCneg Edu- & SMCneg Edu+ & SMCpos Edu- & SMCpos Edu+ & Statistical analysis (Edu-vs. Edu+) \\
\hline $\mathrm{N}$ & 55 & 63 & 29 & 25 & e \\
\hline \multirow[t]{3}{*}{ Age } & $76.2( \pm 0.4 \mathrm{SE})$ & $75.3( \pm 0.3 \mathrm{SE})$ & $77.1( \pm 0.5 \mathrm{SE})$ & $75.9( \pm 0.8 \mathrm{SE})$ & $t$ test: \\
\hline & & & & & SMCneg: n.s. \\
\hline & & & & & SMCpos: n.s. \\
\hline \multirow[t]{3}{*}{$\operatorname{Sex}(M / F)$} & $14 / 41$ & $29 / 34$ & $8 / 21$ & $12 / 13$ & Fisher test: \\
\hline & & & & & SMCneg: n.s. \\
\hline & & & & & SMCpos: n.s. \\
\hline \multirow[t]{3}{*}{ Education } & $4.3( \pm 0.2 \mathrm{SE})$ & $7.8( \pm 0.05 \mathrm{SE})$ & $4.0( \pm 0.2 \mathrm{SE})$ & $7.9( \pm 0.07 \mathrm{SE})$ & $t$ test: \\
\hline & & & & & SMCneg: $p<0.0001$ \\
\hline & & & & & SMCpos: $p<0.0001$ \\
\hline \multirow[t]{3}{*}{ MMSE } & $28.7( \pm 0.11 \mathrm{SE})$ & $29.0( \pm 0.10 \mathrm{SE})$ & $28.6( \pm 0.12 \mathrm{SE})$ & $28.8( \pm 0.16 \mathrm{SE})$ & Mann-Whitney $U$ test: \\
\hline & & & & & SMCneg: n.s. \\
\hline & & & & & SMCpos: n.s. \\
\hline \multirow[t]{6}{*}{ APOE genotype $(\mathrm{N})$} & $\varepsilon 2 / \varepsilon 2(1)$ & $\varepsilon 2 / \varepsilon 2(0)$ & $\varepsilon 2 / \varepsilon 2(0)$ & $\varepsilon 2 / \varepsilon 2(0)$ & $C^{2}$ test $(2 \times 5)$ : SMCneg: n.s. \\
\hline & $\varepsilon 2 / \varepsilon 3$ (10), & ع2/ع3 (7), & ع2/ع3 (2), & ع2/ع3 (2), & $\mathrm{C}^{2}$ test $(2 \times 4):$ SMCpos: n.s. \\
\hline & ع3/ع3 (38) & ع3/ع3 (46) & ع3/ع3 (18) & ع3/ع3 (15) & \\
\hline & $\varepsilon 2 / \varepsilon 4(0)$ & $\varepsilon 2 / \varepsilon 4$ (1) & $\varepsilon 2 / \varepsilon 4(0)$ & $\varepsilon 2 / \varepsilon 4(0)$ & \\
\hline & $\varepsilon 3 / \varepsilon 4$ (6) & $\varepsilon 3 / \varepsilon 4$ (9) & $\varepsilon 3 / \varepsilon 4(8)$ & $\varepsilon 3 / \varepsilon 4(7)$ & \\
\hline & $\varepsilon 4 / \varepsilon 4(0)$ & $\varepsilon 4 / \varepsilon 4(0)$ & $\varepsilon 4 / \varepsilon 4(1)$ & $\varepsilon 4 / \varepsilon 4(1)$ & \\
\hline
\end{tabular}

Edu+ received bachelor, master's degree, or Ph.D. Edu-completed lower education programs

Key: APOE, apolipoprotein E; MMSE, Mini Mental State Examination; M/F, males/females; CSF, cerebrospinal fluid; n.s., not significant ( $p>0.05$ ).

\subsection{Statistical analysis of spectral rsEEG power density}

Three statistical sessions were performed by the commercial tool STATISTICA 10 (StatSoft Inc, www.statsoft.com) to test the study hypotheses. The results (e.g., coefficients and degrees of freedom) were reported using the direct outcome of this tool $(p<0.05)$.

Owing to the factorial design of the present study, we used the linear general model and analysis of variances (ANOVAs) for the comparison of the rsEEG power density variables between the SMC groups and subgroups. However, the use of ANOVA models implies that dependent variables approximate Gaussian distributions while rsEEG power density distributions may not fit this feature. To tackle this issue, we used the following procedure. The Kolmogorov-Smirnov test was used to determine if the rsEEG power density distributions of a given ANOVA model approximated to Gaussian distributions (null hypothesis of non-Gaussian distributions tested at $p<0.05$ ). If this was not the case, all rsEEG power density distributions were processed by the log transformation and re-tested. Such a transformation is a popular method to transform skewed data distribution with all positive values (as rsEEG power density values are) to Gaussian distributions, thus augmenting the reliability of the ANOVA results. The same procedure was used for the sMRI used as inputs for the comparison between SMC groups. The outcome of the procedure approximated all rsEEG power density distributions to Gaussian distributions, allowing the use of the ANOVA models. The same was true for the MRI markers used as inputs for the $t$-tests.

The first statistical session was performed to test the hypothesis that some sMRI markers typically used in AD research might differ

(1) between the SMCneg and SMCpos groups ( $p<0.05$ corrected) and (2) between the Edu- and Edu+ subgroups in both SMCneg and SMCpos seniors $(p<0.05$ corrected). To these aims, four ANOVAs $(p<0.05)$ were performed to compare the following four sMRI makers: the normalized gray matter, white matter, and CSF volume (based on the total intracranial volume), as well as the adjusted hippocampal volume (for the procedure to derive these MRI markers, see Cavedo et al., 2018). The ANOVA factors were education (Edu- and $\mathrm{Edu}+$ ) and group (SMCneg and SMCpos). The degrees of freedom were corrected by the Greenhouse-Geisser procedure when appropriate. Duncan test was used for post hoc comparisons applying a statistical threshold of $p<0.05$ corrected for the planned contrasts. A Bonferroni correction was applied to post hoc solutions for 4 MRI markers $(p<0.05 / 4=0.0125)$ to evaluate statistically significant differences between the SMCneg and SMCpos groups and for 2 SMC subgroups $\times 4$ MRI markers $=8(p<0.05 / 8=0.00625)$ to evaluate statistically significant differences between the Edu- and Edu+ subgroups. The study hypothesis may be confirmed by the following effects: (1) a statistically significant main effect of group factor; (2) a statistically significant ANOVA interaction among the factors Edu and group $(p<0.05)$, and (3) post hoc solutions showing differences between the SMCneg and SMCpos groups $(p<0.0125)$ and between Edu- and Edu+ subgroups for both SMCneg and SMCpos seniors $(p<0.0025)$ in the MRI markers.

The second statistical session was performed to evaluate the hypothesis that the posterior rsEEG power density at the individual alpha 2 and alpha 3 bands might differ (1) between the SMCneg and SMCpos groups and (2) between the Edu- and Edu+ subgroups of the SMCneg and SMCpos seniors $(p<0.05$ corrected). To this aim, an ANOVA used the regional spectral rsEEG power density as a dependent variable. The ANOVA factors were education (Edu- and Edu+), group (SMCneg and SMCpos), band (delta, theta, alpha 1, alpha 2, alpha 3 , beta 1 , beta 2, and gamma), and ROI (frontal, central, parietal, occipital, and temporal). The APOE genotype was used as a covariate. The degrees of freedom were corrected by the Greenhouse-Geisser procedure when appropriate. Duncan test was used for post hoc comparisons using a statistical threshold of $p<0.05$ corrected for the planned contrasts. In line with previous results obtained in ADMCI and AD patients with dementia (Babiloni et al., 2017, 2018), the planned contrasts focused on the comparison of the individual alpha 2 and alpha 3 power density (1) between the SMCneg and SMCpos group and (2) between the Edu- and Edu+ 
subgroups $(p<0.05$ corrected $)$.

Table 3

Mean values $( \pm \mathrm{SE}$ ) of the structural magnetic resonance imaging (MRI) markers, together with the results of their statistical comparisons $(p<0.05)$ in the SMCpos and SMCneg senior groups and the Edu- and Edu+ subgroups

\begin{tabular}{|c|c|c|c|c|c|}
\hline \multicolumn{6}{|l|}{ Mean values ( \pm SE) of MRI data } \\
\hline & \multicolumn{2}{|c|}{ SMCneg } & \multicolumn{2}{|l|}{ SMCpos } & Statistical analysis (SMCneg vs. SMCpos) \\
\hline Normalized gray matter volume & \multicolumn{2}{|c|}{$0.410( \pm 0.003 \mathrm{SE})$} & \multicolumn{2}{|c|}{$0.401( \pm 0.004 \mathrm{SE})$} & Group ANOVA main effect: n.s. \\
\hline Normalized white matter volume & \multicolumn{2}{|c|}{$0.301( \pm 0.002 \mathrm{SE})$} & \multicolumn{2}{|c|}{$0.297( \pm 0.003 \mathrm{SE})$} & Group ANOVA main effect: n.s. \\
\hline Normalized CSF volumeAdjusted & \multicolumn{2}{|c|}{$0.289( \pm 0.003 \mathrm{SE})$} & \multicolumn{2}{|c|}{$0.303( \pm 0.005$} & Group ANOVA main effect: n.s. \\
\hline \multirow[t]{2}{*}{ Adjusted hippocampal volume } & \multicolumn{2}{|c|}{$3,634( \pm 35 \mathrm{SE})$} & \multicolumn{2}{|c|}{$3,460( \pm 50 \mathrm{SE})$} & Group ANOVA main effect: $p<0.007$ \\
\hline & SMCneg Edu- & SMCneg Edu+ & SMCpos Edu- & SMCpos Edu+ & Statistical analysis (Edu-vs. Edu+) \\
\hline Normalized gray matter volume & $0.410( \pm 0.004 \mathrm{SE})$ & $0.409( \pm 0.004 \mathrm{SE})$ & $0.407( \pm 0.005 \mathrm{SE})$ & $0.393( \pm 0.007 \mathrm{SE})$ & $\begin{array}{l}\text { Edu ANOVA main effect or Group*Edu } \\
\text { interaction: } n . s .\end{array}$ \\
\hline Normalized white matter volume & $0.298( \pm 0.003 \mathrm{SE})$ & $0.304( \pm 0.003 \mathrm{SE})$ & $0.295( \pm 0.003 \mathrm{SE})$ & $0.298( \pm 0.005 \mathrm{SE})$ & $\begin{array}{l}\text { Edu ANOVA main effect or Group*Edu } \\
\text { interaction: } n . s .\end{array}$ \\
\hline Normalized CSF volume & $0.291( \pm 0.005 \mathrm{SE})$ & $0.287( \pm 0.005 \mathrm{SE})$ & $0.297( \pm 0.007 \mathrm{SE})$ & $0.309( \pm 0.008 \mathrm{SE})$ & $\begin{array}{l}\text { Edu ANOVA main effect or Group*Edu } \\
\text { interaction: n.s. }\end{array}$ \\
\hline Adjusted hippocampal volume & $3641( \pm 48 \mathrm{SE})$ & $3628( \pm 50 \mathrm{SE})$ & $3469( \pm 53 \mathrm{SE})$ & $3451( \pm 97 \mathrm{SE})$ & $\begin{array}{l}\text { Edu ANOVA main effect or Group*Edu } \\
\text { interaction: n.s. }\end{array}$ \\
\hline
\end{tabular}

Key: CSF, cerebrospinal fluid; n.s., not significant ( $p>0.05$ corrected for multiple comparisons); ANOVA, analysis of variance.

In the above statistical sessions, Bonferroni correction was applied based on the following computation: (1) 2 frequency bands $\mathrm{x} 5$ ROIs $=10(p<0.05 / 10=0.005)$ and (2) 2 SMC groups (SMCneg, SMCpos) x 2 frequency bands x 5 ROIs $=20(p<0.05 / 20=0.0025)$. In this line, the study hypotheses may be confirmed by: (1) a statistically significant ANOVA interaction among the factors group, band, and ROI ( $\mathrm{p}<0.05)$; (2) a statistically significant ANOVA interaction among the factors Edu, group, band, and ROI ( $p<0.05)$; and (3) post hoc solutions showing differences (1) between the SMCneg and SMCpos groups $(p<0.005)$ and (2) between the Edu- and Edu+ subgroups for both SMCneg and SMCpos seniors in the alpha 2 and alpha 3 power density $(p<0.0025)$. Noteworthy, the significant effects of this statistical analysis were controlled by the iterative (leave-one-out) Grubbs' test detecting for the presence of one or more outliers in the distribution of the regional rsEEG power density computed in the Edu- and Edu+ individuals for both SMCneg and SMCpos groups (null hypothesis of non-Gaussian distributions tested at the arbitrary threshold of $p<0.001$, to remove only individual values with high probability to be outliers and maintain the random sampling of the SMC population with the rsEEG features described above).

The third statistical session was performed to correlate two relevant neuropsychological scores to the posterior alpha 2 and alpha 3 power density across the SMCpos and SMCneg groups considered separately $(p<0.05$ corrected). The selected neuropsychological variables were the MMSE score (as a rough index of global cognition) and the duration of delayed copy of Rey figure (30 minutes; as an index of episodic memory performance). As a composite rsEEG marker, the posterior alpha power density was calculated as the mean of parietal, occipital, and temporal alpha power density values. Spearman test was used for the correlation of EEG variables with MMSE scores, while Pearson test was used for the correlation with scores of Copy of Rey Figure test. A Bonferroni correction was applied equal to 2 neuropsychological scores $\times 2$ frequency bands $=4(p<0.05 / 4=0.0125)$.

\section{Results}

\subsection{Characterization of brain structural integrity in the SMCneg and SMCpos groups and effect of education}

Table 3 reports the mean values $( \pm$ SE) of the following four relevant sMRI markers for the Edu- and Edu+ subgroups in the SMCneg and SMCpos seniors: the normalized gray matter volume, normalized white matter volume, normalized CSF volume, and the adjusted hippocampal volume. Four ANOVAs were performed having these MRI markers as dependent variables and group (SMCneg, SMCpos) and education (Edu-, Edu+) as factors. The results of the comparison of these markers between the SMCneg and SMCpos groups (Bonferroni corrected: $p<0.0125$ ) and between the Edu+ and Edu- subgroups (Bonferroni corrected: $p<0.00625$ ) are reported in the following. A statistically significant main effect of the group factor was found only for the adjusted hippocampal volume $(\mathrm{F}[1,143]=7.7, p<0.007)$, which was lower in the SMCpos than the SMCneg group. Furthermore, there was a statistical trend in the main effect of the group factor for the normalized CSF volume. Specifically, there was a larger normalized CSF volume in the SMCpos than the SMCneg group ( $p<0.05$ uncorrected).

No statistically significant difference or trends were found between the Edu- and Edu+ subgroups $(p>0.00625)$.

\subsection{Quality control of the regional alpha power density spectra}

Fig. 1 shows the mean values $( \pm$ SE, log transformed) of the regional rsEEG power density spectra in the SMCneg and SMCpos groups. In both groups, the posterior (i.e., parietal, temporal, and occipital) regional rsEEG power density spectra were characterized by well-shaped alpha peaks and other benchmark features of rsEEG recordings of sufficient quality such as (1) visible TFs; (2) drop of rsEEG power density values as a function of the increase of rsEEG frequencies after alpha power density peaks; (3) no significant power density offsets at gamma frequencies in some ROIs; and (4) no multiple rsEEG power density peaks in the spectra.

\subsection{Comparisons in the regional alpha power density between the SMCneg and SMCpos groups and effect of education}

Fig. 2 shows the mean values $( \pm$ SE, log transformed) of the regional rsEEG power density spectra for the Edu- and Edu+ subgroups in the SMCneg and SMCpos seniors. Compared with the SMCneg group, the SMCpos group exhibited similar magnitude in the 
rsEEG power density across the spectra. No statistically significant effect was produced by the ANOVA $(p>0.05)$ including the factors group (SMCneg, SMCpos), band (delta, theta, alpha 1, alpha 2, beta 1, beta 2, and gamma), and ROI (frontal, central, parietal, occipital and temporal).

The ANOVA showed a statistically significant 3-way interaction effect $(\mathrm{F}(28,4704)=2.5, p<0.00005)$ among the factors group (SMCneg, SMCpos), education (Edu-, Edu+), band (delta, theta, alpha 1, alpha 2, beta 1, beta 2, and gamma), and ROI (frontal, central, parietal, occipital and temporal). The Duncan planned post hoc test $(p<0.0025$, equivalent to $p<0.05$ corrected $)$ showed that compared with the SMCpos Edu- subgroup, the SMCpos Edu+ subgroup pointed to (1) lower occipital alpha 2 power density ( $p<$

0.000005) and (2) higher temporal alpha 3 power density $(p<0.0001)$. Opposite effects of the education level were observed in the SMCneg seniors. Compared with the SMCneg Edu- subgroup, the SMCneg Edu+ subgroup exhibited higher parietal $(p<0.00001)$, occipital $(p<0.000005)$, and temporal $(p<0.00001)$ alpha 2 power density as well as higher parietal $(p<0.0001)$, occipital $(p<$ $0.000005)$, and temporal $(p<0.0001)$ alpha 3 power density. The above effects of the education level were because of neither the presence of outliers (statistical threshold of Grubbs' test at $p<0.001$; Fig. 3) nor a different number of artifact-free rsEEG epochs included in the analysis (no statistically significant differences between Edu- and Edu+ groups according to $t$ test, $p>0.05$ ). In the results of this last section, the most statistically significant effects of the education level were observed for the occipital alpha 2 power density in both SMCneg and SMCpos subgroups $(p<0.000005)$. Therefore, we tested the hypothesis that the education level may be related to differences in the following relevant variables between the SMCneg and SMCpos subgroups: immediate copy of Rey figure, delayed copy of Rey figure (30 minutes), MRI markers of occipital thickness, and occipital 18F-florbetapir PET SUVR. Specifically, the following comparisons were performed for the above neuropsychological, MRI, and PET variables separately: SMCneg Edu- versus SMCneg Edu+ and SMCpos $\mathrm{Edu}^{-}$versus SMCpos Edu+ ( $t$ test at $p<0.00625$, equivalent to $p<0.05$ corrected for eight comparisons). In the SMCneg subgroups, the only remarkable effect was a statistical trend in the delayed copy of the Rey figure ( $p<0.05$ uncorrected), indicating a better score in the SMCneg Edu+ subgroup compared with the SMCneg Edu- sub-group. In the SMCpos subgroups, no statistically significant effect was observed ( $p>0.1$ uncorrected).

\subsection{Correlation between posterior alpha power density and neuropsychological scores in the SMCneg and SMCpos groups}

In the SMCneg seniors, there was a statistically significant $(p<0.0125$ equivalent to $p<0.05$ corrected) positive correlation between the posterior alpha 2 power density and the MMSE score $(r=0.23, p=0.01)$. The higher the alpha 2 power density, the higher the MMSE score. In the same seniors, just a trend ( $p<0.05$ uncorrected) was observed in the positive correlation between the posterior alpha 3 power density and the MMSE score $(\mathrm{r}=0.21, p=0.02)$.

SMCneg vs SMCpos

Mean values $( \pm S E)$ of normalized rsEEG power density

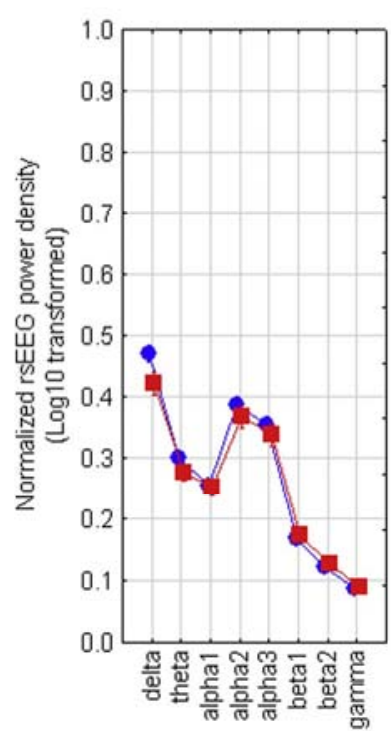

Frontal

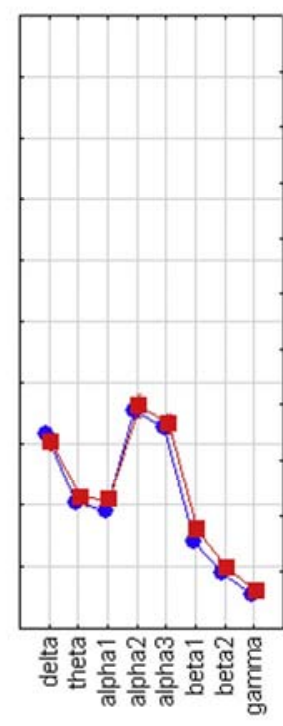

Central

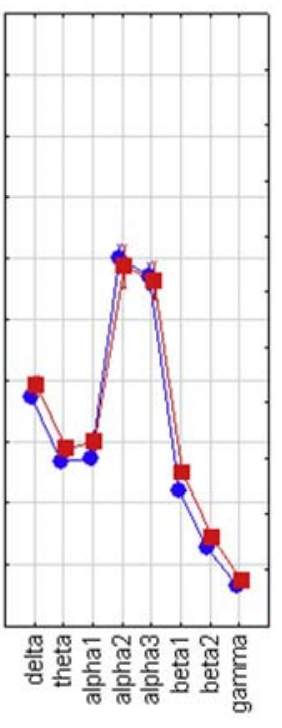

Parietal

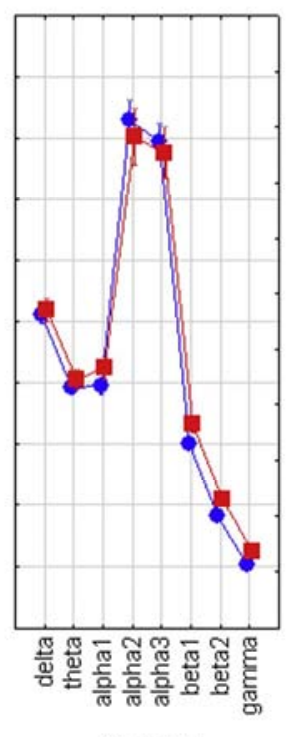

Occipital

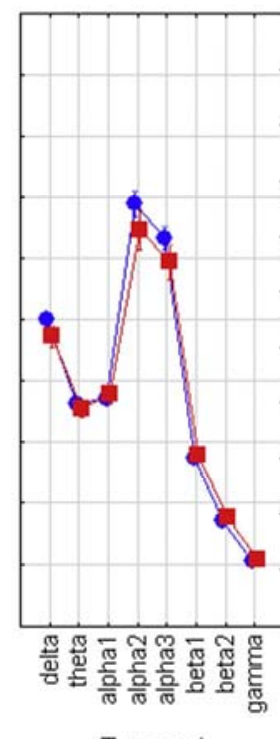

Temporal
SMCneg SMCpos

Fig. 1. Mean values ( \pm standard error mean, SE) of power density in normalized resting state eyes-closed electroencephalographic (rsEEG) rhythms in seniors with subjective memory complaint (SMC) found to be amyloid-negative (SMCneg) and amyloid-positive (SMCpos) to the marker of Alzheimer's neuropathology derived from ${ }^{18} \mathrm{~F}$-florbetapir positron emission tomography (amyPET). These data are ordered for (1) two groups (SMCneg and SMCpos seniors), (2) five regions of interest (ROIs) of the scalp (frontal, central, parietal, occipital, and temporal), and (3) eight frequency bands of rsEEG rhythms (delta, theta, alpha 1, alpha 2, alpha 3, beta 1, beta 2, and gamma). No statistically significant effect was produced by the ANOVA design $(p>0.05)$ using the factors group, band, and ROI. Abbreviations: ANOVA, analysis of variance.

In the SMCpos seniors, there was no statistically significant correlation between the posterior alpha 2 and alpha 3 power density and the MMSE score $(p>0.7)$.

Finally, no statistically significant correlation was found between the posterior alpha power density and the score of delayed Copy of Rey Figure test ( $p>0.1$ for SMCneg and $p>0.7$ for SMCpos seniors). 
A cross-validation analysis was performed to evaluate whether the above effects of the education level on the regional rsEEG power density, based on a ${ }^{18} \mathrm{~F}$-florbetapir PET SUVR threshold of 0.79 , was confirmed using the more conservative ${ }^{18} \mathrm{~F}$-florbetapir PET SUVR threshold of 0.88 . The latter was used in the quoted study investigating functional brain connectivity from rsEEG alpha and beta rhythms in the INSIGHT-preAD cohort (Teipel et al., 2017a,b).

In this cross-validation analysis, the SMC seniors were grouped as follows: 63 SMCneg Edu- (15 male; mean age: 76.5 years; mean education level: 4.3; mean MMSE score: 28.7), 71 SMCneg Edu+ (31 male; mean age: 75.3 years; mean education level: 7.8; mean MMSE score: 29.0), 21 SMCpos Edu- (7 male; mean age: 76.6 years; mean education level: 4.0; mean MMSE score: 28.6), and 17 SMCpos Edu+ (10 male; mean age: 76.2 years; mean education level: 7.8 ; mean MMSE score: 28.7 ).

No statistically significant difference was found for the age, sex, and MMSE score between the Edu- and Edu+ subgroups in the SMCneg and SMCpos seniors considered separately ( $p>0.05$ uncorrected in all comparisons; see the tests of the principal analysis).

The ANOVA and Duncan post hoc tests evaluating the effects of the education level on the regional rsEEG power density showed the same effects of the principal analysis, so cross validating its results. Specifically, there was a statistically significant 3 -way interaction effect $(\mathrm{F}[28,4676]=2.6, p<0.00001)$ among the factors education (Edu-, Edu+), group (SMCneg, SMCpos), band (delta, theta, alpha 1, alpha 2, beta 1, beta 2, and gamma), and ROI (frontal, central, parietal, occipital and temporal). The Duncan planned post hoc test ( $p$ $<0.0025$, equivalent to $p<0.05$ corrected as in the principal analysis) showed that compared with the SMCpos Edu- subgroup, the SMCpos Edu+ subgroup pointed to lower parietal $(p<0.00005)$ and occipital $(p<0.000005)$ alpha 2 power density. Opposite effects were observed in the SMCneg subgroups. Compared with the SMCneg Edu- subgroup, the SMCneg Edu+ subgroup exhibited higher parietal $(p<0.0001)$, occipital $(p<0.000005)$, and temporal $(p<0.00001)$ alpha 2 power density as well as occipital $(p<0.00001)$ and temporal $(p<0.0001)$ alpha 3 power density.

The above effects were not because of the presence of outliers (statistical threshold of Grubbs' test at $p<0.001$ ). 
SMCneg

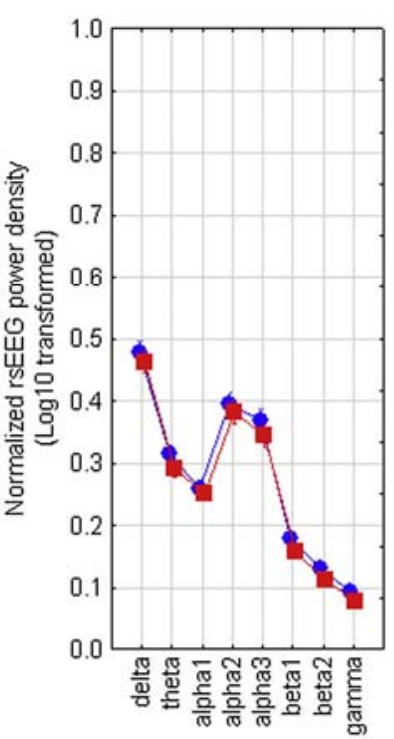

Frontal

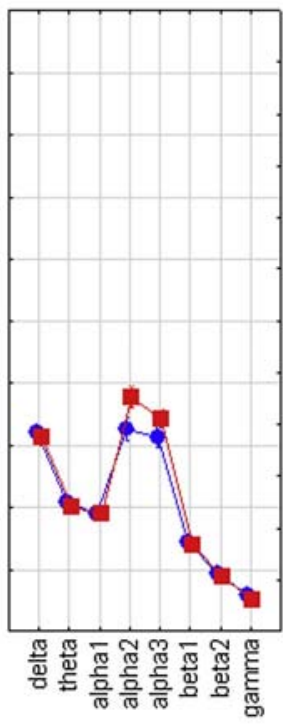

Central

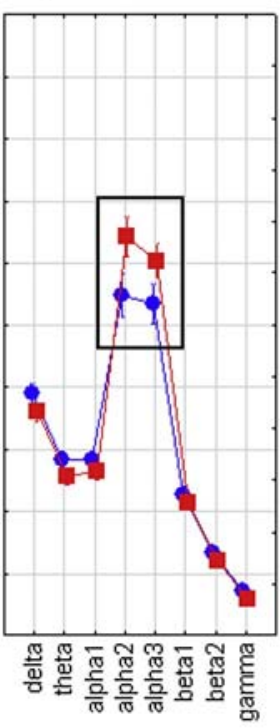

Parietal

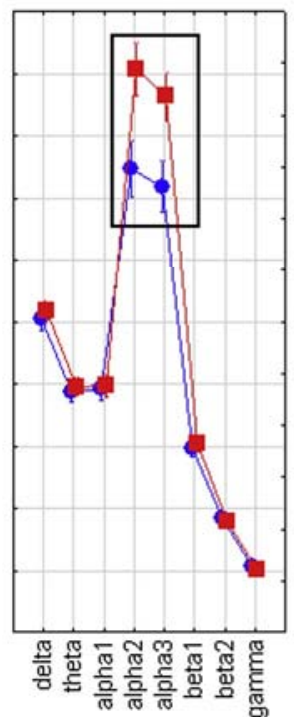

Occipital

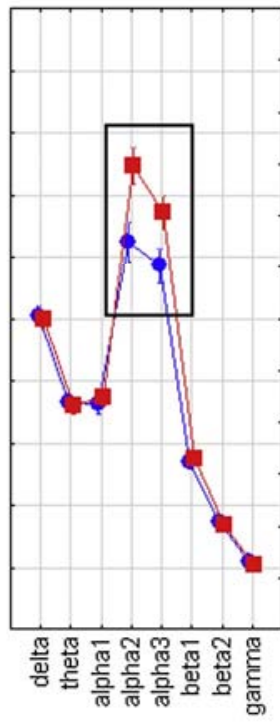

Temporal
Edu-

Edu+

SMCpos

Mean values $( \pm S E)$ of normalized rsEEG power density
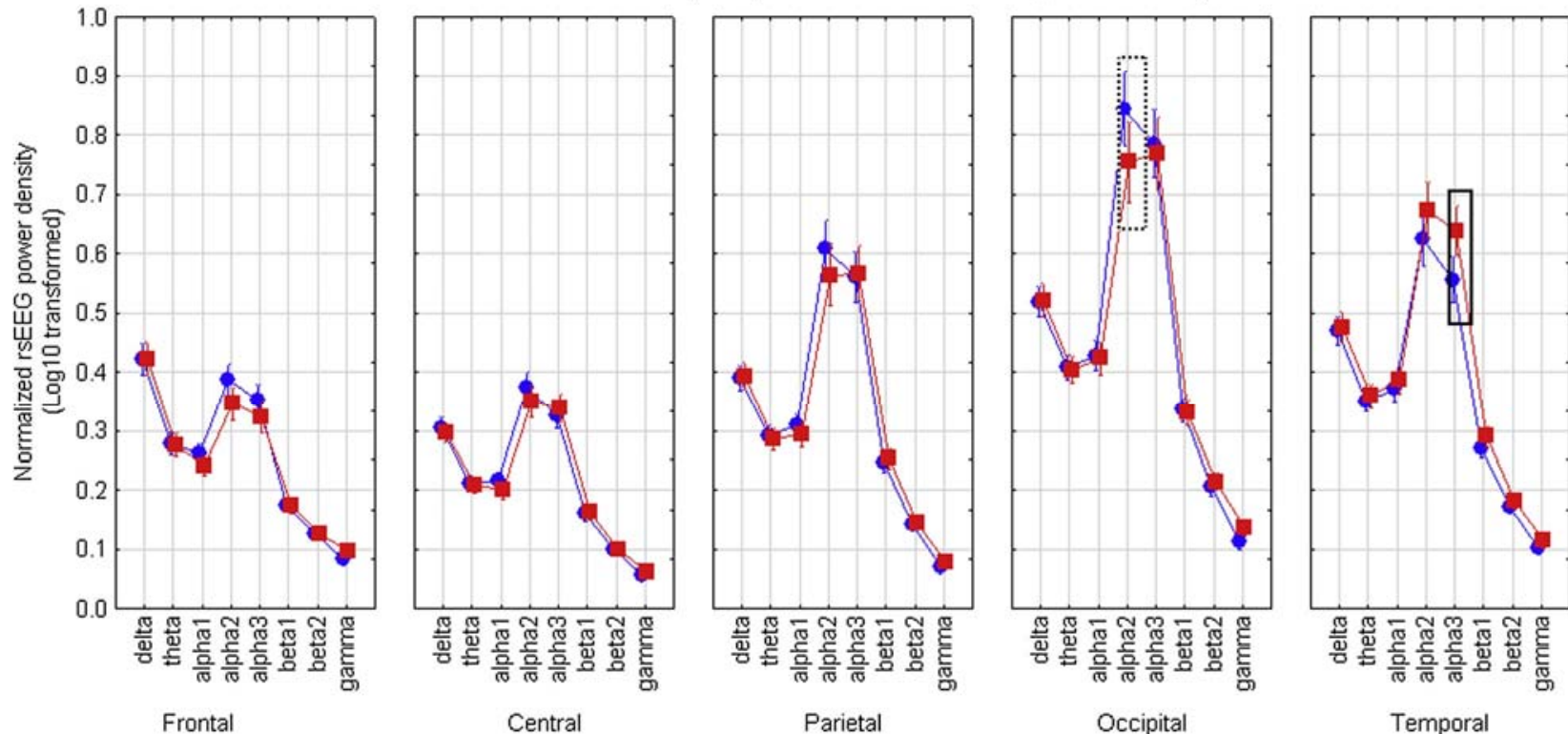

EduEdu+

Fig. 2. Mean values ( \pm SE) of normalized rsEEG power density for (1) two groups (SMCneg and SMCpos seniors), (2) two education levels (Edu+ and Edu-), (3) five ROIs (frontal, central, parietal, occipital, and temporal), and (4) eight frequency bands (delta, theta, alpha 1, alpha 2, alpha 3, beta 1, beta 2, and gamma). The ANOVA showed a statistically significant 3-way interaction effect $(\mathrm{F}[28,4676]=2.5, p<0.00005)$ among the factors group, education, band, and ROI. The rectangles indicate the scalp regions and frequency bands in which the rsEEG power density presented statistically significant pattern: Edu $+\mathbf{s}$ Edu-in the SMCneg and SMCpos senior groups (Duncan post hoc test, $p<0.05$ corrected for multiple comparisons $=p<0.0025$; see "Methods" for the criterion of such correction). Abbreviations: rsEEG, resting state eyes-closed electroencephalographic; SMC, subjective memory complaint; ROIs, regions of interest; ANOVA, analysis of variance. 

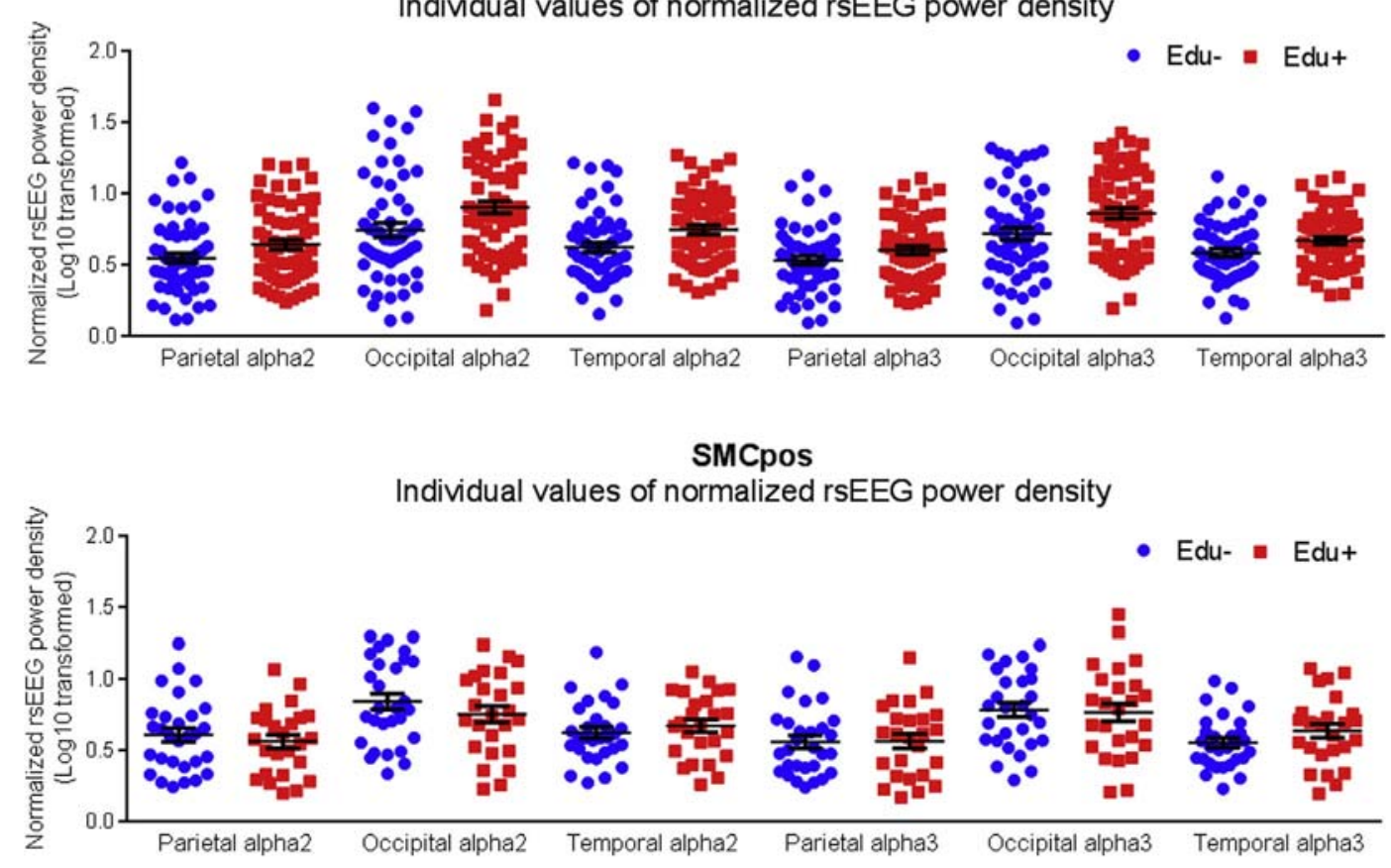

Fig. 3. Individual values of the normalized rsEEG power density showing statistically significant $(p<0.05$ corrected for multiple comparisons $=p<0.0025)$ differences between the groups of SMCpos and SMCneg seniors stratified according to the high (Edu+) or low (Edu-) educational level (i.e., parietal alpha 2, occipital alpha 2, temporal alpha 2, parietal alpha 3, occipital alpha 3, and temporal alpha 3). Noteworthy, the Grubbs' test showed no outliers from those individual values of the normalized rsEEG power densities (arbitrary threshold at $p<0.001$; see "Methods" for the criterion of such correction). Abbreviations: rsEEG, resting state eyes-closed electroencephalographic; SMC, subjective memory complaint.

\subsection{Control analysis}

As a secondary (control) cross-validation rsEEG analysis, we used a data driven approach without constraints in a priori frequency bands (i.e., delta to gamma) and scalp regions of interest (i.e., occipital to frontal lobes). For this analysis, we used a Fieldtrip software plugin (Oostenveld et al., 2011), available through the Study tool of the interactive EEGLAB package (Delorme and Makeig, 2004). The original plugin implements a nonparametric Monte Carlo method using a statistical permutation by which the significance probability returned by the randomization test is a Monte Carlo estimate of the $p$-value under the permutation distribution (Nichols and Holmes, 2001). To address the problem of multiple comparisons for EEG frequencies and electrodes, cluster-correction statistics were chosen, based on clustering of adjacent time or frequency-samples exhibiting similar differences (Maris and Oostenveld, 2007). Cluster-based correction within the sensor and frequency dimensions was applied by defining clusters through the selection of neighboring electrodes with the triangulation method (based on a 2D projection of the sensor positions) and taking the maximum of the cluster-level statistics (a cluster-level statistic is equal to the sum of the sample-specific t-statistics that belong to this cluster; Maris and Oostenveld, 2007). The threshold of the corrected $p$ value was set to 0.05 .

As an add-on variant of the original plugin (not available on default options), we implemented the normalization of power density estimates before the mentioned statistical analysis. This normalization was done by an external MATLAB scrip integrated with the study tool available in the EEGLAB package. This script normalized EEG power density estimates by the mean value of EEG power density evaluated across all electrodes and frequency bins from 0.5 to $45 \mathrm{~Hz}$. Unfortunately, this secondary (control) analysis could not take into account the individual variation of alpha frequency peak (i.e., IAFp), not implemented in the Fieldtrip toolbox used.

In the secondary (control) analysis, two statistical sessions were performed. The first session compared the EEG (normalized) power density between Edu- and Edu+ subgroups in the SMCpos seniors, whereas the second session compared it between the Edu- and Edu+ subgroups in the SMCneg seniors.

Results of this analysis were as follows. After the permutation test, no statistically significant effect was observed in the first session $(p>0.05$ corrected).

In contrast, one statistically significant effect was observed in the second session $(p<0.05$ corrected). Compared with the SMCneg group with Edu-, the SMCneg group with Edu+ showed higher (normalized) alpha power density at T5 electrode (i.e., left temporal region) from 4 to $17 \mathrm{~Hz}$ ( $p<0.05$ corrected), namely an effect centered around $10 \mathrm{~Hz}$ (alpha). For illustrative purposes, Fig. 4 shows the topographic maps of the (normalized) spectral power density in the SMCneg Edu+ (left) and Edu- (middle) groups at both 9.5 Hz (upper row) and $10 \mathrm{~Hz}$ (lower row) frequency bins, which exhibited the most significant statistical differences between the two subgroups $(p<0.01$ uncorrected). The results of this analysis were also represented in Fig. 5. This Figure plots the rsEEG (normalized) power density spectra from 0.5 to $45 \mathrm{~Hz}$ at T5 electrode in the SMCneg Edu+ (left) and Edu- (middle) subgroups. It also plots a diagram showing the frequency range from 4 to $17 \mathrm{~Hz}$ in which the two rsEEG (normalized) power density spectra differed ( $p<0.05$ corrected). Overall, these results are consistent with those reported in the main analysis showing greater normalized power density at temporal alpha rhythms in the SMCneg Edu+ subgroup compared with the SMCneg Edu- (middle) subgroup. 


\section{Discussion}

In previous investigations, abnormalities in posterior rsEEG rhythms were reported in ADMCI and ADD compared with seniors with intact cognition (Dierks et al., 1993, 2000; Jelic et al., 2000; Jeong, 2004; Ponomareva et al., 2003) as well as patients with dementia and MCI due to Parkinson's and Lewy Body diseases (Babiloni et al., 2017, 2018). These previous findings made posterior alpha rhythms an interesting marker to test the effect of high education attainment in preclinical AD stages. In this line, the present retrospective study tested the hypothesis that abnormalities of those alpha rhythms may characterize SMC seniors with preclinical Alzheimer's neuropathology (SMCpos) as a function of the education level.

\subsection{Resting state alpha rhythms do not change in SMCpos seniors as a whole}

Compared with the SMCneg, the SMCpos showed neither differences in posterior alpha rhythms nor in the other frequency bands. These findings were found with the more liberal ${ }^{18} \mathrm{~F}$-flor-betapir PET SUVR threshold at 0.79 and were confirmed with the one at 0.88 (not shown). Therefore, the present results do not extend to the present whole group of SMCpos seniors the abnormalities in posterior alpha rhythms previously reported in ADMCI and ADD (Babiloni et al., 2017, 2018).

As such, the present results agree with the evidence of two previous rsEEG studies performed in the INSIGHT-preAD cohort (Teipel et al., 2017a; Gaubert et al., 2019). In the first rsEEG study (Teipel et al., 2017a), 318 SMC seniors were divided in two groups, 63 SMCpos and 255 SMCneg individuals based on the ${ }^{18} \mathrm{~F}$-florbetapir PET SUVR threshold of 0.88 . The rsEEG-dependent variables included markers of power density, phase lag index, and spectral coherence computed from 70 selected scalp electrodes. The fixed frequency bands were delta $(2-3.9 \mathrm{~Hz})$, theta $(4-7.3 \mathrm{~Hz})$, low alpha $(7.5-9.75 \mathrm{~Hz})$, high alpha $(10.25-12.5 \mathrm{~Hz})$, beta $(13-30 \mathrm{~Hz})$, and gamma $(31-49$ $\mathrm{Hz})$. Results showed no statistical associations between global ${ }^{18} \mathrm{~F}$-florbetapir PET SUVR and those EEG variables in the whole group of SMC seniors. However, when only SMCpos seniors were considered, there was a statistical trend (noncorrected for statistical comparisons) for higher global ${ }^{18} \mathrm{~F}$-florbetapir PET SUVR and lower functional connectivity (phase lag index) of parietal and temporal alpha and beta rhythms.

\section{Normalized rsEEG power density}

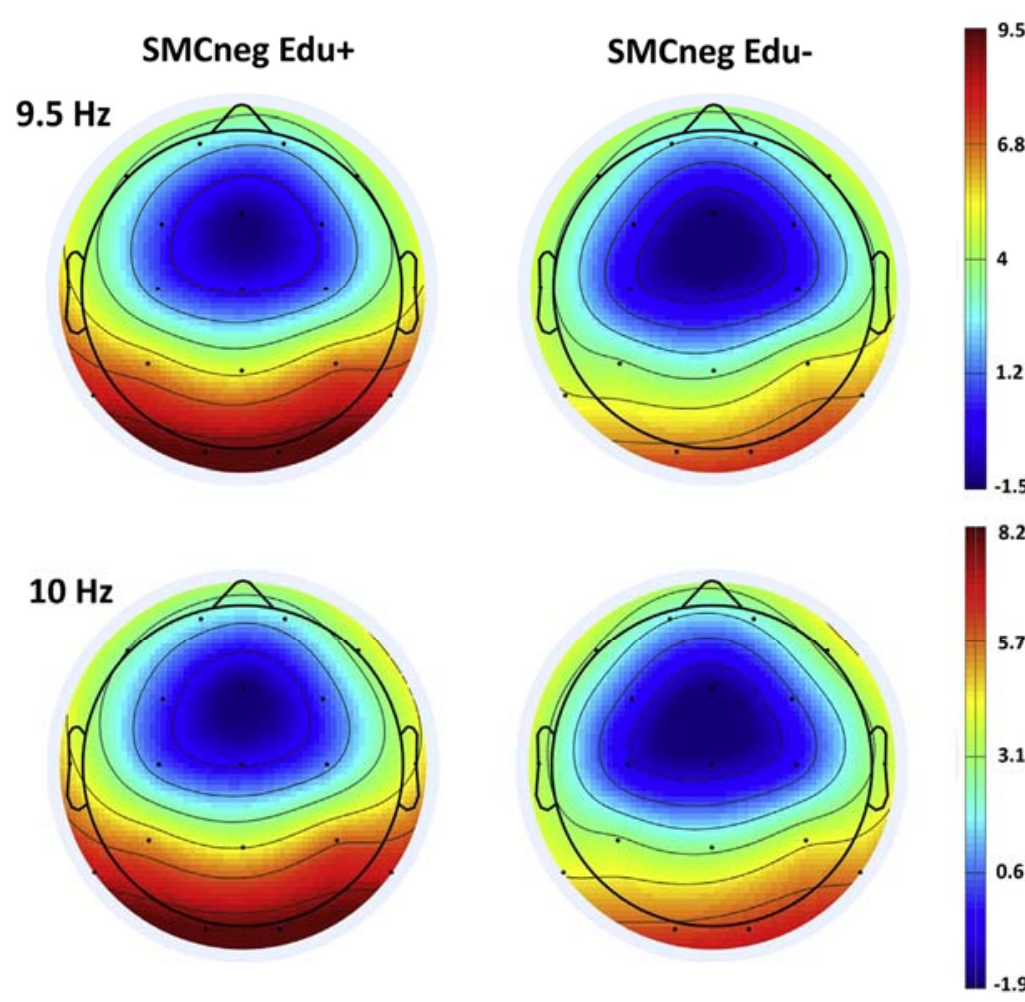

\section{p-values}
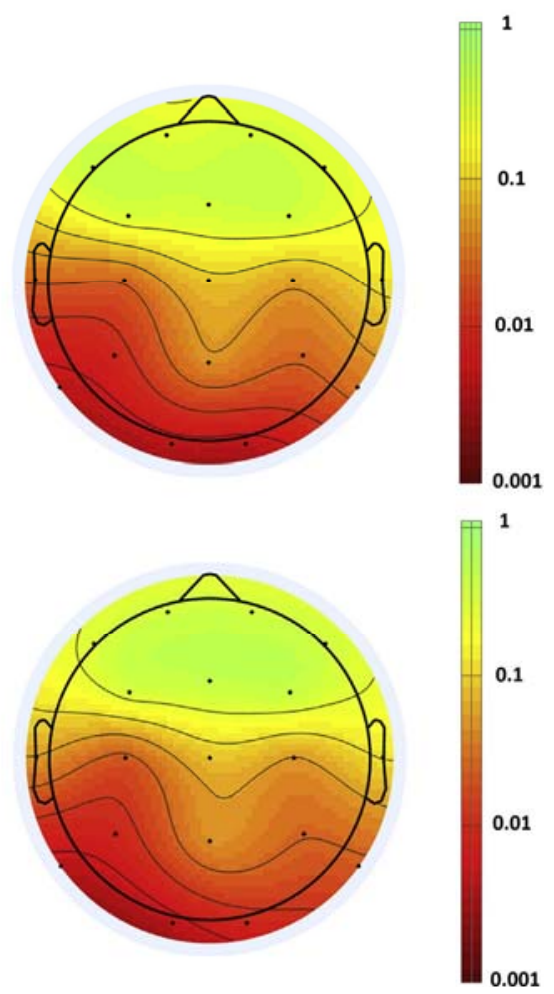

Fig. 4. Topographical maps of the normalized spectral rsEEG power density computed from 19 electrodes of 10-20 system in the SMCneg Edu+ (left) and Edu- (middle) subgroups of older adults. The computation was performed at $9.5 \mathrm{~Hz}$ (upper row) and $10 \mathrm{~Hz}$ (lower row) frequency bins, which were those expressing the most statistically significant differences between the two subgroups (red color indicates higher values of spectral power density). Right column reports the topographical maps with $p$ values (uncorrected) computed through a Monte Carlo permutation test assessing the statistical differences in alpha power density between the SMCneg Edu- and Edu+ subgroups (red color indicates lower $p$-values). (For interpretation of the references to color in this figure legend, the reader is referred to the Web version of this article.) Abbreviations: rsEEG, resting state eyesclosed electroencephalographic; SMC, subjective memory complaint. 


\section{Individual normalized rsEEG power density at T5 electrode}
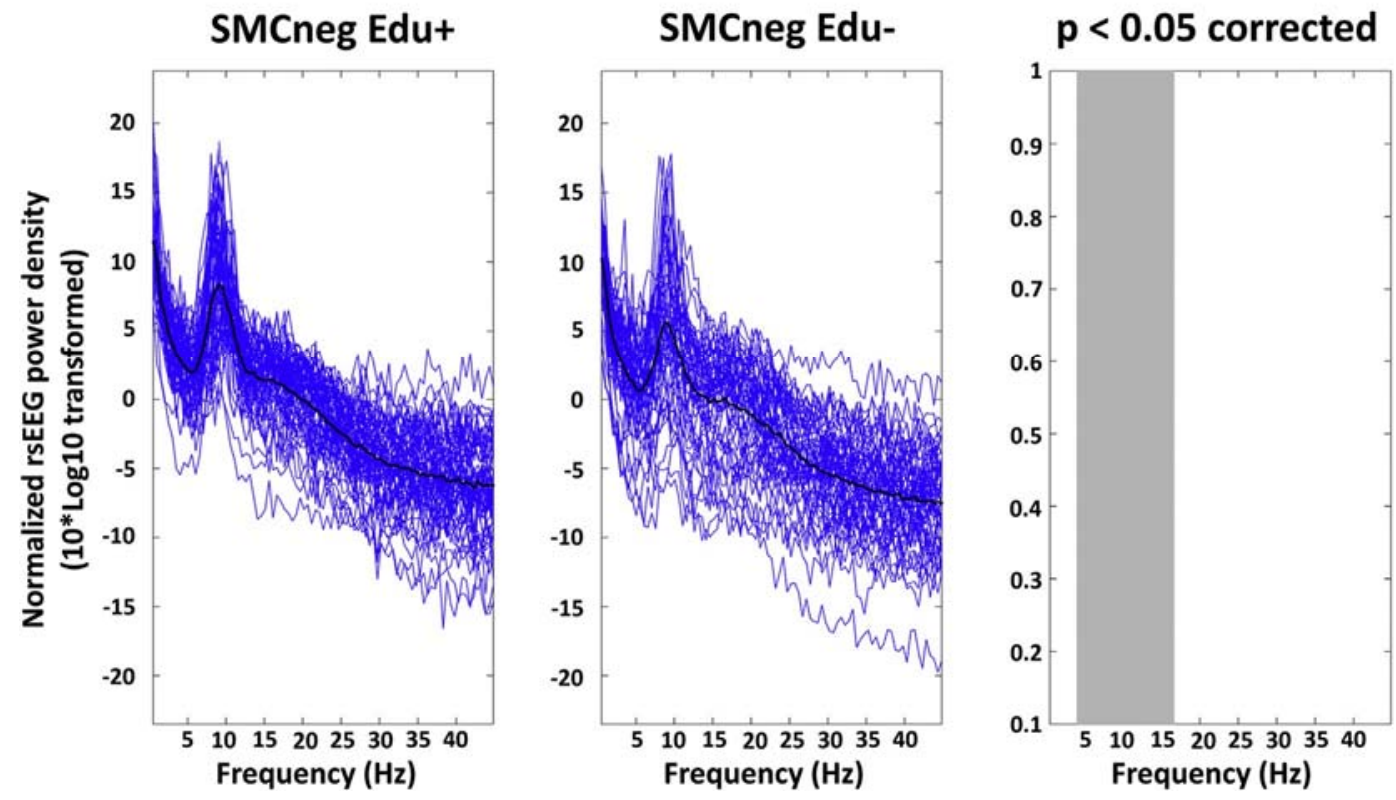

Fig. 5. The left and middle panels plot individual normalized rsEEG power density spectra (blue lines) computed at T5 electrode of 10-20 system in the SMCneg Edu+ and Edusubgroups of older adults in the frequency range from 0.5 to $45 \mathrm{~Hz}$. In those panels, the normalized spectral rsEEG power density values averaged across all individuals within each subgroup (black thick lines) are also plotted. The right panel graphically indicates the frequency bins showing statistically significant differences ( $p<0.05$ corrected) in rsEEG power density between the two SMCneg subgroups of older adults (those significant frequencies meet the gray rectangle). (For interpretation of the references to color in this figure legend, the reader is referred to the Web version of this article.) Abbreviations: rsEEG, resting state eyes-closed electroencephalographic; SMC, subjective memory complaint.

In the second rsEEG study (Gaubert et al., 2019), 25 SMCpos positive to FDG-PET, 63 SMCpos negative to FDG-PET, 51 SMCneg positive to FDG-PET, and 175 SMCneg negative to FDG-PET were grouped based on the ${ }^{18} \mathrm{~F}$-florbetapir PET SUVR threshold of 0.79 . The rsEEG dependent variables at single electrodes included power density, median spectral frequency, spectral entropy measure, and signal complexity estimates based on information theory. Another information-theoretic measurement estimated the interdependence of rsEEG rhythms at pairs of electrodes (i.e., one electrode against all the others). The fixed frequency bands of interest were delta (1-4

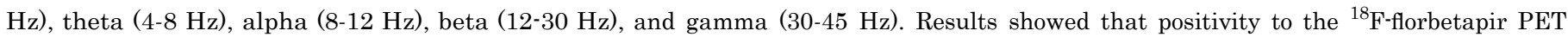
SUVR (i.e., indicative of Alzheimer's neuropathology) and rsEEG variables had a nonlinear relationship, namely a U-shape curve with delta power density and inverted U-shape with beta, gamma, median spectral frequency, spectral entropy, and complexity more obvious for intermediate to very high amyloid load individuals. As mentioned above, amyloid burden did not show noticeable effects on alpha, and the association between FDG-PET and alpha was not clear.

Taken together, the results of the two previous INSIGHT-preAD studies (Teipel et al., 2017a; Gaubert et al., 2019) and the present one, which were based on different methodologies (i.e., two

different ${ }^{18} \mathrm{~F}$-florbetapir PET thresholds; fixed and individual alpha

frequency bands; linear and non-linear metrics; rsEEG power density and between-electrodes interdependence, etc.), do converge on the conclusion that the resting-state posterior alpha rhythms may not substantially reflect preclinical Alzheimer's neuropathology in SMCpos seniors as a whole group.

The above lack of effects may not be because of unfavorable signal-to-noise ratio in the rsEEG data or interindividual variability in alpha frequencies. Indeed, here we implemented and use the following ad hoc procedure to tackle those confounders (see Methods), which produced final rsEEG power density spectra with the following benchmarks of good quality: (1) posterior regional rsEEG power density spectra with well-shaped TF and alpha peaks;

(2) rsEEG power density values progressively dropping after the alpha peak in the frequency range; and (3) no remarkable offset in the rsEEG power density at gamma frequencies in the temporal and frontal ROIs (such offset is typically induced by EMG artifacts because of head and neck muscle tensions).

\subsection{Resting state alpha rhythms do change in SMC seniors with high education level}

As a novelty, here we report that compared with the SMCpos and SMCneg seniors with low-moderate education level, those with high education level (i.e., bachelor, master's degree or $\mathrm{PhD}$ title) exhibited significant changes in amplitude of alpha rhythms in posterior areas of the scalp. This effect was obtained with both relevant $18 \mathrm{~F}$-florbetapir PET SUVR thresholds (0.79 and 0.88) in the stratification of SMC individuals and may be specific in frequency. In fact, the other rsEEG frequency bands including delta and theta exhibited no significant effects. Furthermore, differences in alpha rhythms did not depend on cognitive status and abilities of daily living as the subgroups were carefully matched for those variables. More specifically, results showed that posterior alpha rhythms were (beneficially) higher in amplitude in the SMCneg senior sub-group (i.e., without preclinical Alzheimer's neuropathology) with high over low-moderate education level. This difference might unveil a neuroprotective effect of the high education level on neurophysiological oscillatory mechanisms modulating dominant alpha rhythms in quiet vigilance, when $\mathrm{AD}$ neuropathology is not challenging the brain and CR may be still available. In this line, previous sMRI evidence showed that SMC seniors with high over low education level were characterized by larger cortical thickness (Vaqué-Alcázar et al., 2017), which may mitigate brain white matter abnormalities and delay MCI onset over time (Mortamais et al., 2014). Noteworthy, this neuroprotective effect may resist in temporal cortex even for a certain load of Alzheimer's 
neuropathology. Indeed, the present SMCpos senior subgroups (i.e., with preclinical Alzheimer's neuropathology) showed a similar effect in temporal areas. Specifically, comparing with the SMCpos senior subgroup with low-moderate education level that with high education level showed greater amplitude in temporal alpha rhythms. At this early stage of the research, it was unclear why this effect may be limited to temporal cortical areas possibly generating those rhythms. Future studies should test if the well-known high metabolic rate of neurons in medial temporal cortex and hippocampus may play a role in explaining the present findings.

In the SMCpos seniors (i.e., with preclinical Alzheimer's neuropathology), the picture was quite different in occipital areas. In fact, occipital alpha rhythms showed lower (not higher) amplitude in the SMCpos seniors with high over low-moderate education level. Noteworthy, this effect was not associated with greater occipital atrophy, occipital amyloid- $B$ accumulation, and worse scores in the Rey figure copy (visual function). The present results in the SMCpos seniors complement previous neuroimaging evidence in AD patients. In sMRI studies carried out in ADMCI, a high CR (i.e., years of education, reading, and vocabulary measures, etc.) was associated with the following findings: (1) reduced cortical thickness values and later progression to dementia (Pettigrew et al., 2017; Querbes et al., 2009); (2) decreased left hippocampus volume and cortical thickness in the right supramarginal gyrus (Arenaza-Urquijo et al., 2013); and (3) decreased volume in the medial prefrontal, anterior cingulate, and orbitofrontal areas (Bartrés-Faz et al., 2019). In ADD, high education level was related to smaller regional cortical thickness in frontal, temporal, and parietal association areas in one sMRI study (Seo et al., 2011) and in temporal gyrus, inferior and superior parietal gyri, and lateral occipital cortex in another sMRI study (Liu et al., 2012).

In the same line, previous FDG-PET studies of resting-state neuronal metabolic activity unveiled that a high education level was related to hypometabolism in temporo-parietal and ventral prefrontal brain areas in seniors with intact cognition and $\mathrm{AD}$ preclinical neuropathology (Ewers et al., 2013), whereas it was related to hypometabolism in the precuneus (Franzmeier et al., 2017) or a set of regions including posterior parietal, posterior cingulate, and precuneus regions (Bauckneht et al., 2018) in ADMCI. Interestingly, a significant Alzheimer's disease neuroimaging initiative study showed that the education level might not predict amyloid$B$ deposition or brain metabolism rate in ADMCI, so the CR might not affect the progression in the Alzheimer's neuropathology (Wada et al., 2018).

Keeping in mind the above data and considerations, we posit that the present amplitude reduction in occipital alpha rhythms in SMCpos seniors with high education level might be because of altered synchronization mechanisms in neural circuits impinging upon thalamic nuclei and posterior sagittal cortex before the appearance of substantial local AD neurodegeneration and cognitive deficits. This lack of consequences of the alterations in alpha rhythms may be because of a compensatory residual brain reserve factor represented in those circuits, including posterior nodes of default mode network. According to previous functional MRI studies in healthy older adults (Franzmeier et al., 2018a), ADMCI (Franzmeier et al., 2017a) and ADD (Franzmeier et al., 2018b) with high educational attainment may be characterized by an increase of functional connectivity between a frontal network spanning prefrontal and premotor areas and other cortical circuits involved in general cognition and episodic memory such as default mode and dorsal frontoparietal attention networks impinging upon posterior sagittal cortex. Therefore, such an increase of functional connectivity may enhance the efficiency of those networks not only in frontal but also posterior cortical areas, attenuating the positive relationship between $\mathrm{AD}$ neuropathology-neurodegeneration and clinical manifestation of the disease typically observed in $\mathrm{AD}$ patients with low education attainment (Franzmeier etal., 2017; Stern et al., 2018).

At this early stage of the research, we do not know the relationship among amyloid- $\beta$ deposition in the brain, the mentioned decrease in amplitude of occipital alpha rhythms dominant in quiet wakefulness, and brain correlates of high education level in SMC seniors with Alzheimer's neuropathology. It can be speculated that such an amyloid- $B$ deposition may induce thalamus-cortical overexciting synchronization signals reflected by the reduction of dominant occipital alpha rhythms in quiet wakefulness (those rhythms mainly underpin inhibition of cortical primary and associative sensory areas in the experimental conditions of absence of sensory stimulations as resting state is; Pfurtscheller and Lopes da Silva, 1999). This speculation is based on the following data and considerations. Previous studies have shown that posterior alpha rhythms in quiet wakefulness were lower in amplitude in MCI patients positive over negative to AD markers of AB42 and phospho tau in the CSF (Jovicich et al., 2019). Furthermore, increased cortical neuronal hyperactivity and excitability generating epileptiform activity may be associated with earlier clinical onset of AD and faster disease progression (Amatniek et al., 2006; Cretin et al., 2016; de Waal et al., 2011; Horvath et al., 2016, 2018; Vossel et al., 2016). In the same line of "overexcitation" hypothesis, transgenic mouse models of AD with APP and/or PS1

mutations showed brain amyloid plaque deposition and neurodegeneration, cognitive deficits, alterations in on-going dominant EEG oscillatory activity recorded in quiet wakefulness, and epileptiform activity (Del Percio et al., 2018; Gurevicius et al., 2013; Micotti et al., 2015; Palop et al., 2009).

\subsection{Methodological remarks}

In the interpretation of the present findings, the following methodological limitations should be considered.

This retrospective study was based on data collected by the only French clinical unit involved in the INSIGHT-preAD project. As such, the study ensured the maximum methodological consistency in the SMC seniors' enrollment and data collection. However, the study results will require cross-validation by a multicenter clinical trial with independent and "harmonized" clinical units.

Owing to the intense experimental plan, just 1 minute of rsEEG recordings at eyes closed was available. Results of the present study encourage investments for future rsEEG recordings lasting some minutes in the continuation of the INSIGHT-preAD project.

The topographical analysis of rsEEG rhythms has been done using only 19 scalp electrodes of traditional 10-20 montage system to ensure the best control of EEG signal and comparability of the present findings with those of previous rsEEG studies carried out in AD patients by the Authors (Babiloni et al., 2010, 2017, 2018; Galluzzi et al., 2016; Jovicich et al., 2019). As a rationale of this methodological option, a bulk of previous studies showed that (eyes closed) rsEEG rhythms are characterized by low-frequency spatial contents well sampled by 10-20 montage system. Indeed, they are formed by a sequence of short (i.e., few seconds), quasi-stable topographical patterns of EEG voltage maps with large centroids of voltage positive and negative maxima (several centimeters), the socalled brain electrical microstates (Lehmann et al., 1987). Abnormalities in the mentioned microstates may be associated with alterations in a set of distributed cortical neural networks spanning frontal, parietal, temporal, occipital, and limbic areas (Khanna et al., 2015; Michel and Koenig, 2018). Keeping in mind these data and considerations, we think that the present topographical analysis of rsEEG rhythms could successfully account for (1) subject-wise individual features of posterior alpha rhythms in quiet wakefulness, generated by distributed neural networks, and (2) spatial abnormalities in posterior alpha rhythms detected in AD patients even at the disease stage of SMC in relation to the education attainment, in line with a recent position paper of Electrophysiology Professional Interest Area of International Alzheimer's Association (Babiloni et al., 2020). 


\section{Conclusions}

In the present study, we tested whether SMC seniors associated with Alzheimer's neuropathology (SMCpos) and high education level may present abnormal resting state posterior rsEEG rhythms at alpha (8e12 Hz) frequencies, as predicted by the general theory of the CR (Stern et al., 2018). The SMCneg participants with high (over low-moderate) education level showed the higher amplitude of posterior alpha rhythms (possibly "neuroprotective"). Furthermore, the SMCpos participants with high (over low-moderate) education level showed the higher amplitude of temporal alpha rhythms (possibly "neuroprotective") and lower amplitude of posterior alpha rhythms (possibly "compensatory"). This effect may reflect CR as no differences in brain gray-white matter and cognitive functions were observed between these SMCpos/SMCneg subgroups. Overall, preclinical Alzheimer's neuropathology may interact with education attainment and neurophysiological mechanisms generating cortical alpha rhythms in quiet wakefulness.

These results and conclusions motivate future multicenter studies in SMCpos seniors using harmonized protocols and data collection hardware for the cross-validation of the present findings. Those studies may test the hypothesis that as a function of the high education level, posterior alpha rhythms in quiet wakefulness may provide useful first-level biomarkers in prevention programs developed in SMC seniors to undergo to more invasive or expensive diagnostic procedures of $\mathrm{AD}$, especially in lower-income and middle-income countries and underserved populations.

\section{Disclosure statement}

None of the authors has relevant conflicts of interest to disclose.

\section{CRediT authorship contribution statement}

Claudio Babiloni: Conceptualization, Methodology, Formal analysis, Validation, Writing - original draft, Supervision, Writing - review \& editing. Susanna Lopez: Conceptualization, Methodology, Formal analysis, Validation, Writing - original draft, Supervision, Writing review \& editing. Claudio Del Percio: Formal analysis, Software, Writing - review \& editing. Giuseppe Noce: Formal analysis, Software, Writing - review \& editing. Maria Teresa Pascarelli: Formal analysis, Software, Writing - review \& editing. Roberta Lizio: Formal analysis, Software, Writing - review \& editing. Stefan J. Teipel: Investigation, Data curation, Project administration, Writing review \& editing. Gabriel González-Escamilla: Investigation, Data curation, Project administration, Writing - review \& editing. Hovagim Bakardjian: Investigation, Data curation, Project administration, Writing - review \& editing. Nathalie George: Investigation, Data curation, Project administration, Writing - review \& editing. Enrica Cavedo: Investigation, Data curation, Project administration, Writing - review \& editing. Simone Lista: Investigation, Data curation, Project administration, Writing - review \& editing. Patrizia Andrea Chiesa: Investigation, Data curation, Project administration, Writing - review \& editing. Andrea Vergallo: Investigation, Data curation, Project administration, Writing - review \& editing. Pablo Lemercier: Investigation, Data curation, Project administration, Writing - review \& editing. Giuseppe Spinelli: Investigation, Data curation, Project administration, Writing - review \& editing. Michel J. Grothe: Investigation, Data curation, Project administration, Writing - review \& editing. Marie-Claude Potier: Investigation, Data curation, Project administration, Writing - review \& editing. Fabrizio Stocchi: Investigation, Data curation, Project administration, Writing - review \& editing. Raffaele Ferri: Investigation, Data curation, Project administration, Writing - review \& editing. MarieOdile Habert: Investigation, Data curation, Project administration, Writing - review \& editing. Francisco J. Fraga: Formal analysis, Software, Writing - review \& editing. Bruno Dubois: Investigation, Data curation, Project administration, Writing - review \& editing. Harald Hampel: Investigation, Data curation, Project administration, Writing - review \& editing.

\section{Acknowledgements}

The present study was developed based on the data of the INSIGHT-preAD project.

The study was promoted by INSERM in collaboration with the Institut du Cerveau et de la Moelle Epiniere (ICM), Institut des Neurosciences Translationnelles de Paris (IHU-A-ICM), Pfizer and Institut de la Mémoire et de la Maladie d'Alzheimer (IM2A) who supported the INSIGHT-preAD project as well as the 318 INSIGHT-preAD volunteers The study was promoted also in collaboration with the "CHU de Bordeaux" (coordination CIC EC7), the promoter of Memento cohort, funded by the Alzheimer Foundation Plan. The study was further supported by AVID/Lilly. The authors thank Christiane Metzinger (ICM) for INSIGHT data management.

The INSIGHT-preAD project and the research activities of the neuroimaging platform of the ICM have received funding from the program "Investissements d'avenir" ANR-10-IAIHU-06 and ANR-11-INBS-0006'.

In this study, the electroencephalographic data analysis was partially supported by the funds of "Ricerca Corrente" attributed by Italian Ministry of Health to the IRCCS SDN of Naples, IRCCS OASI of Troina, and IRCCS San Raffaele Pisana of Rome.

Scientific activity of Prof. Francisco J. Fraga was developed thanks to the financing received within the grant \#2018/03655-1 from Sao Paulo Research Foundation (FAPESP).

\section{References}

Albert, M.S., DeKosky, S.T., Dickson, D., Dubois, B., Feldman, H.H., Fox, N.C., Gamst, A., Holtzman, D.M., Jagust, W.J., Petersen, R.C., Snyder, P.J., Carrillo, M.C., Thies, B., Phelps, C.H., 2011. The diagnosis of mild cognitive impairment due to Alzheimer's disease: recommendations from the National Institute on Aging-Alzheimer's Association Workgroups on diagnostic guidelines for Alzheimer's disease. Alzheimers Dement. 7, 270e279.

Alexander, D.M., Arns, M.W., Paul, R.H., Rowe, D.L., Cooper, N., Esser, A.H., Fallahpour, K., Stephan, B.C., Heesen, E., Breteler, R., Williams, L.M., Gordon, E., 2006. EEG markers for cognitive decline in elderly subjects with subjective memory complaints. J. Integr. Neurosci. 5, 49e74.

Amatniek, J.C., Hauser, W.A., DelCastillo-Castaneda, C., Jacobs, D.M., Marder, K., Bell, K., Albert, M., Brandt, J., Stern, Y., 2006. 
Incidence and predictors of seizures in patients with Alzheimer's disease. Epilepsia 47, 867e872.

Amieva, H., Mokri, H., Le Goff, M., Meillon, C., Jacqmin-Gadda, H., Foubert-Samier, A., Orgogozo, J.M., Stern, Y., Dartigues, J.F., 2014. Compensatory mechanisms in higher-educated subjects with Alzheimer's disease: a study of 20 years of cognitive decline. Brain 137 (Pt 4), 1167e1175.

Arenaza-Urquijo, E.M., Molinuevo, J.L., Sala-Llonch, R., Solé-Padullés, C., Balasa, M., Bosch, B., Olives, J., Antonell, A., Lladó, A., Sánchez-Valle, R., Rami, L., Bartrés-Faz, D., 2013. Cognitive reserve proxies relate to gray matter loss in cognitively healthy elderly with abnormal cerebrospinal fluid amyloid-B levels. J. Alzheimers Dis. 35, 715e726.

Babiloni, C., Binetti, G., Cassetta, E., Dal Forno, G., Del Percio, C., Ferreri, F., Ferri, R., Frisoni, G., Hirata, K., Lanuzza, B., Miniussi, C., Moretti, D.V., Nobili, F., Rodriguez, G., Romani, G.L., Salinari, S., Rossini, P.M., 2006a. Sources of cortical rhythms change as a function of cognitive impairment in pathological aging: a multi-centric study. Clin. Neurophysiol. 117, $252 \mathrm{e} 268$.

Babiloni, C., Cassetta, E., Dal Forno, G., Del Percio, C., Ferreri, F., Ferri, R., Lanuzza, B., Miniussi, C., Moretti, D.V., Nobili, F., Pascual-Marqui, R.D., Rodriguez, G., Luca Romani, G., Salinari, S., Zanetti, O., Rossini, P.M., 2006b. Donepezil effects on sources of cortical rhythms in mild Alzheimer's disease: Responders vs. non-responders. Neuroimage 31, $1650 \mathrm{e} 1665$.

Babiloni, C., Benussi, L., Binetti, G., Bosco, P., Busonero, G., Cesaretti, S., Dal Forno, G., Del Percio, C., Ferri, R., Frisoni, G., Ghidoni, R., Rodriguez, G., Squitti, R., Rossini, P.M., 2006c. Genotype (cystatin C) and EEG phenotype in Alzheimer disease and mild cognitive impairment: a multicentric study. Neuroimage 29, 948e964.

Babiloni, C., Pievani, M., Vecchio, F., Geroldi, C., Eusebi, F., Fracassi, C., Fletcher, E., De Carli, C., Boccardi, M., Rossini, P.M., Frisoni, G.B., 2009. White-matter lesions along the cholinergic tracts are related to cortical sources of EEG rhythms in amnesic mild cognitive impairment. Hum. Brain Mapp. 30, 1431e1443.

Babiloni, C., Visser, P.J., Frisoni, G., De Deyn, P.P., Bresciani, L., Jelic, V., Nagels, G., Rodriguez, G., Rossini, P.M., Vecchio, F., Colombo, D., Verhey, F., Wahlund, L.O., Nobili, F., 2010. Cortical sources of resting EEG rhythms in mild cognitive impairment and subjective memory complaint. Neurobiol. Aging 31, 1787e1798.

Babiloni, C., Carducci, F., Lizio, R., Vecchio, F., Baglieri, A., Bernardini, S., Cavedo, E., Bozzao, A., Buttinelli, C., Esposito, F., Giubilei, F., Guizzaro, A., Marino, S., Montella, P., Quattrocchi, C.C., Redolfi, A., Soricelli, A., Tedeschi, G., Ferri, R., RossiFedele, G., Ursini, F., Scrascia, F., Vernieri, F., Pedersen, T.J., Hardemark, H.G., Rossini, P.M., Frisoni, G.B., 2013. Resting state cortical electroencephalographic rhythms are related to gray matter volume in subjects with mild cognitive impairment and Alzheimer's disease. Hum. Brain Mapp. 34, 1427e1446.

Babiloni, C., Del Percio, C., Boccardi, M., Lizio, R., Lopez, S., Carducci, F., Marzano, N., Soricelli, A., Ferri, R., Triggiani, A.I., Prestia, A., Salinari, S., Rasser, P.E., Basar, E., Famà, F., Nobili, F., Yener, G., Emek-Savaş, D.D., Gesualdo, L., Mundi, C., Thompson, P.M., Rossini, P.M., Frisoni, G.B., 2015. Occipital sources of resting-state alpha rhythms are related to local gray matter density in subjects with amnesic mild cognitive impairment and Alzheimer's disease. Neurobiol. Aging 36, 556e570.

Babiloni, C., Del Percio, C., Caroli, A., Salvatore, E., Nicolai, E., Marzano, N., Lizio, R., Cavedo, E., Landau, S., Chen, K., Jagust, W., Reiman, E., Tedeschi, G., Montella, P., De Stefano, M., Gesualdo, L., Frisoni, G.B., Soricelli, A., 2016. Cortical sources of resting state EEG rhythms are related to brain hypometabolism in subjects with Alzheimer's disease: an EEG-PET study. Neurobiol. Aging 48, $122 \mathrm{e} 134$.

Babiloni, C., Del Percio, C., Lizio, R., Noce, G., Cordone, S., Lopez, S., Soricelli, A., Ferri, R., Pascarelli, M.T., Nobili, F., Arnaldi, D., Aarsland, D., Orzi, F., Buttinelli, C., Giubilei, F., Onofrj, M., Stocchi, F., Stirpe, P., Fuhr, P., Gschwandtner, U., Ransmayr, G., Caravias, G., Garn, H., Sorpresi, F., Pievani, M., Frisoni, G.B., D’Antonio, F., De Lena, C., Güntekin, B., Hanoglu, L., Baş ar, E., Yener, G., Emek-Savaş, D.D., Triggiani, A.I., Franciotti, R., De Pandis, M.F., Bonanni, L., 2017. Abnormalities of cortical neural synchronization mechanisms in patients with dementia due to Alzheimer's and Lewy body diseases: an EEG study. Neurobiol. Aging 55, 143e158.

Babiloni, C., Del Percio, C., Lizio, R., Noce, G., Lopez, S., Soricelli, A., Ferri, R., Pascarelli, M.T., Catania, V., Nobili, F., Arnaldi, D., Famà, F., Aarsland, D., Orzi, F., Buttinelli, C., Giubilei, F., Onofrj, M., Stocchi, F., Vacca, L., Stirpe, P., Fuhr, P., Gschwandtner, U., Ransmayr, G., Garn, H., Fraioli, L., Pievani, M., Frisoni, G.B., D’Antonio, F., De Lena, C., Güntekin, B., Hanoglu, L., Baş ar, E., Yener, G., Emek-Savaş, D.D., Triggiani, A.I., Franciotti, R., Taylor, J.P., De Pandis, M.F., Bonanni, L., 2018. Abnormalities of resting state cortical EEG rhythms in subjects with mild cognitive impairment due to Alzheimer's and Lewy body diseases. J. Alzheimers Dis. $62,247 \mathrm{e} 268$.

Babiloni, C., Blinowska, K., Bonanni, L., Cichocki, A., De Haan, W., Del Percio, C., Dubois, B., Escudero, J., Fernández, A., Frisoni, G., Guntekin, B., Hajos, M., Hampel, H., Ifeachor, E., Kilborn, K., Kumar, S., Johnsen, K., Johannsson, M., Jeong, J., LeBeau, F., Lizio, R., Lopes da Silva, F., Maestú, F., McGeown, W.J., McKeith, I., Moretti, D.V., Nobili, F., Olichney, J., Onofrj, M., Palop, J.J., Rowan, M., Stocchi, F., Struzik, Z.M., Tanila, H., Teipel, S., Taylor, J.P., Weiergräber, M., Yener, G., Young-Pearse, T., Drinkenburg, W.H., Randall, F., 2020. What electrophysiology tells us about Alzheimer's disease: a window into the synchronization and connectivity of brain neurons. Neurobiol. Aging 85, 58e73.

Bartrés-Faz, D., González-Escamilla, G., Vaqué-Alcázar, L., Abellaneda-Pérez, K., Valls-Pedret, C., Ros, E., Grothe, M.J., 2019. Characterizing the molecular Architecture of cortical regions associated with high educational attainment in older individuals. J. Neurosci. 39, 4566e4575.

Barulli, D., Stern, Y., 2013. Efficiency, capacity, compensation, maintenance, plasticity: emerging concepts in cognitive reserve. Trends Cogn. Sci. 17, 502e509.

Bauckneht, M., Chincarini, A., Piva, R., Arnaldi, D., Girtler, N., Massa, F., Pardini, M., Grazzini, M., Efeturk, H., Pagani, M., Sambuceti, G., Nobili, F., Morbelli, S., 2018. Metabolic correlates of reserve and resilience in MCI due to Alzheimer's Disease (AD). Alzheimers Res. Ther. 10, 35.

Bennett, D.A., Wilson, R.S., Schneider, J.A., Evans, D.A., Mendes de Leon, C.F., Arnold, S.E., Barnes, L.L., Bienias, J.L., 2003. Education modifies the relation of AD pathology to level of cognitive function in older persons. Neurology 60, $1909 \mathrm{e} 1915$.

Brassen, S., Adler, G., 2003. Short-term effects of acetylcholinesterase inhibitor treatment on EEG and memory performance in Alzheimer patients: an open, controlled trial. Pharmacopsychiatry 36, 304e308.

Buchan, R.J., Nagata, K., Yokoyama, E., Langman, P., Yuya, H., Hirata, Y., Hatazawa, J., Kanno, I., 1997. Regional correlations between the EEG and oxygen metabolism in dementia of Alzheimer's type. Electroencephalogr. Clin. Neurophysiol. $103,409 \mathrm{e} 417$.

Cavedo, E., Chiesa, P.A., Houot, M., Ferretti, M.T., Grothe, M.J., Teipel, S.J., Lista, S., Habert, M.O., Potier, M.C., Dubois, B., Hampel, H., INSIGHT-preAD Study Group, Alzheimer Precision Medicine Initiative (APMI), 2018. Sex differences in functional and 
molecular neuroimaging biomarkers of Alzheimer's disease in cognitively normal older adults with subjective memory complaints. Alzheimers Dement. 14, 1204e1215.

Chaves, M.L., Camozzato, A.L., Köhler, C., Kaye, J., 2010. Predictors of the progression of dementia severity in brazilian patients with Alzheimer's disease and vascular dementia. Int. J. Alzheimers Dis. 2010.

Clark, C.M., Pontecorvo, M.J., Beach, T.G., Bedell, B.J., Coleman, R.E., Doraiswamy, P.M., Fleisher, A.S., Reiman, E.M., Sabbagh, M.N., Sadowsky, C.H., Schneider, J.A., Arora, A., Carpenter, A.P., Flitter, M.L., Joshi, A.D., Krautkramer, M.J., Lu, M., Mintun, M.A., Skovronsky, D.M., AV-45-A16 Study Group, 2012. Cerebral PET with florbetapir compared with neuropathology at autopsy for detection of neuritic amyloid-B plaques: a prospective cohort study. Lancet Neurol. 11, $669 \mathrm{e} 678$.

Cretin, B., Sellal, F., Philippi, N., Bousiges, O., Di Bitonto, L., Martin-Hunyadi, C., Blanc, F., 2016. Epileptic prodromal Alzheimer's disease, a retrospective study of 13 new cases: expanding the spectrum of Alzheimer's disease to an epileptic variant? J. Alzheimers Dis. $52,1125 \mathrm{e} 1133$.

de Waal, H., Stam, C.J., Blankenstein, M.A., Pijnenburg, Y.A., Scheltens, P., van der Flier, W.M., 2011. EEG abnormalities in early and late onset Alzheimer's disease: understanding heterogeneity. J. Neurol. Neurosurg. Psychiatry 82, 67e71.

Del Percio, C., Drinkenburg, W., Lopez, S., Limatola, C., Bastlund, J.F., Christensen, D.Z., Pedersen, J.T., Forloni, G., Frasca, A., Noe, F.M., Bentivoglio, M., Fabene, P.F., Bertini, G., Colavito, V., Dix, S., Ferri, R., Bordet, R., Richardson, J.C., Babiloni, C., 2018. Ongoing electroencephalographic activity associated with cortical arousal in transgenic PDAPP mice (hAPP V717F). Curr. Alzheimer Res. 15, 259e272.

Delorme, A., Makeig, S., 2004. EEGLAB: an open source toolbox for analysis of single-trial EEG dynamics including independent component analysis. J. Neurosci. Methods 134, 9e21.

Devanand, D., Lee, J., Luchsinger, J., Manly, J., Marder, K., Mayeux, R., Scarmeas, N., Schupf, N., Stern, Y., 2013. Lessons from epidemiologic research about risk factors, modifiers, and progression of late onset Alzheimer's Disease in New York City at Columbia University Medical Center. J. Alzheimers Dis. 33 (Suppl 1), S447eS455.

Dierks, T., Ihl, R., Frölich, L., Maurer, K., 1993. Dementia of the Alzheimer type: effects on the spontaneous EEG described by dipole sources. Psychiatry Res. 50, $151 \mathrm{e} 162$.

Dierks, T., Jelic, V., Pascual-Marqui, R.D., Wahlund, L., Julin, P., Linden, D.E., Maurer, K., Winblad, B., Nordberg, A., 2000. Spatial pattern of cerebral glucose metabolism (PET) correlates with localization of intracerebral EEG-generators in Alzheimer's disease. Clin. Neurophysiol. 111, $1817 \mathrm{e} 1824$.

Dubois, B., Feldman, H.H., Jacova, C., Hampel, H., Molinuevo, J.L., Blennow, K., DeKosky, S.T., Gauthier, S., Selkoe, D., Bateman, R., Cappa, S., Crutch, S., Engelborghs, S., Frisoni, G.B., Fox, N.C., Galasko, D., Habert, M.O., Jicha, G.A., Nordberg, A., Pasquier, F., Rabinovici, G., Robert, P., Rowe, C., Salloway, S., Sarazin, M., Epelbaum, S., de Souza, L.C., Vellas, B., Visser, P.J., Schneider, L., Stern, Y., Scheltens, P., Cummings, J.L., 2014. Advancing research diagnostic criteria for Alzheimer's disease: the IWG-2 criteria. Lancet Neurol. 13, 614e629.

Dubois, B., Epelbaum, S., Nyasse, F., Bakardjian, H., Gagliardi, G., Uspenskaya, O., Houot, M., Lista, S., Cacciamani, F., Potier, M.C., Bertrand, A., Lamari, F., Benali, H., Mangin, J.F., Colliot, O., Genthon, R., Habert, M.O., Hampel, H., INSIGHT-preAD Study Group, 2018. Cognitive and neuroimaging features and brain B-amyloidosis in individuals at risk of Alzheimer's disease (INSIGHT-preAD): a longitudinal observational study. Lancet Neurol. 17, $335 \mathrm{e} 346$.

Ewers, M., Insel, P.S., Stern, Y., Weiner, M.W., Alzheimer's Disease Neuroimaging Initiative (ADNI), 2013. Cognitive reserve associated with FDG-PET in preclinical Alzheimer disease. Neurology 80, 1194e1201.

Franzmeier, N., Göttler, J., Grimmer, T., Drzezga, A., Áraque-Caballero, M.A., Simon-Vermot, L., Taylor, A.N.W., Bürger, K., Catak, C., Janowitz, D., Müller, C., Duering, M., Sorg, C., Ewers, M., 2017a. Resting-state connectivity of the left frontal cortex to the default mode and dorsal attention network supports reserve in mild cognitive impairment. Front. Aging Neurosci. 9, 264.

Franzmeier, N., Duering, M., Weiner, M., Dichgans, M., Ewers, M., Alzheimer's Disease Neuroimaging Initiative (ADNI), 2017b. Left frontal cortex connectivity underlies cognitive reserve in prodromal Alzheimer disease. Neurology 88, $1054 \mathrm{e} 1061$.

Franzmeier, N., Hartmann, J., Taylor, A.N.W., Araque-Caballero, M.Á., Simon-Vermot, L., Kambeitz-Ilankovic, L., Bürger, K., Catak, C., Janowitz, D., Müller, C., Ertl-Wagner, B., Stahl, R., Dichgans, M., Duering, M., Ewers, M., 2018a. The left frontal cortex supports reserve in aging by enhancing functional network efficiency. Alzheimers Res. Ther. $10,28$.

Franzmeier, N., Düzel, E., Jessen, F., Buerger, K., Levin, J., Duering, M., Dichgans, M., Haass, C., Suárez-Calvet, M., Fagan, A.M., Paumier, K., Benzinger, T., Masters, C.L., Morris, J.C., Perneczky, R., Janowitz, D., Catak, C., Wolfsgruber, S., Wagner, M., Teipel, S., Kilimann, I., Ramirez, A., Rossor, M., Jucker, M., Chhatwal, J., Spottke, A., Boecker, H., Brosseron, F., Falkai, P., Fliessbach, K., Heneka, M.T., Laske, C., Nestor, P., Peters, O., Fuentes, M., Menne, F., Priller, J., Spruth, E.J., Franke, C., Schneider, A., Kofler, B., Westerteicher, C., Speck, O., Wiltfang, J., Bartels, C., Araque Caballero, M.Á., Metzger, C., Bittner, D., Weiner, M., Lee, J.H., Salloway, S., Danek, A., Goate, A., Schofield, P.R., Bateman, R.J., Ewers, M., 2018b. Left frontal hub connectivity delays cognitive impairment in autosomal-dominant and sporadic Alzheimer's disease. Brain 141, $1186 \mathrm{e} 1200$.

Galluzzi, S., Marizzoni, M., Babiloni, C., Albani, D., Antelmi, L., Bagnoli, C., Bartres-Faz, D., Cordone, S., Didic, M., Farotti, L., Fiedler, U., Forloni, G., Girtler, N., Hensch, T., Jovicich, J., Leeuwis, A., Marra, C., Molinuevo, J.L., Nobili, F., Pariente, J., Parnetti, L., Payoux, P., Del Percio, C., Ranjeva, J.P., Rolandi, E., Rossini, P.M., Schönknecht, P., Soricelli, A., Tsolaki, M., Visser, P.J., Wiltfang, J., Richardson, J.C., Bordet, R., Blin, O., Frisoni, G.B., PharmaCog Consortium, 2016. Clinical and biomarker profiling of prodromal Alzheimer's disease in workpackage 5 of the Innovative Medicines Initiative PharmaCog project: a 'European ADNI study. J. Intern. Med. 279, 576e591.

Gaubert, S., Raimondo, F., Houot, M., Corsi, M.C., Naccache, L., Diego Sitt, J., Hermann, B., Oudiette, D., Gagliardi, G., Habert, M.O., Dubois, B., De Vico Fallani, F., Bakardjian, H., Epelbaum, S., Alzheimer's Disease Neuroimaging Initiative, MEMENTO Study Group, INSIGHT-preAD Study Group, 2019. EEG evidence of compensatory mechanisms in preclinical Alzheimer's disease. Brain 142, 2096e2112.

Gonzalez-Escamilla, G., Atienza, M., Cantero, J.L., 2015. Impaired cortical oscillatory coupling in mild cognitive impairment: anatomical substrate and ApoE4 effects. Brain Struct. Funct. 220, 1721e1737.

Gonzalez-Escamilla, G., Atienza, M., Garcia-Solis, D., Cantero, J.L., 2016. Cerebral and blood correlates of reduced functional connectivity in mild cognitive impairment. Brain Struct. Funct. 221, 631e645.

Gouw, A.A., Alsema, A.M., Tijms, B.M., Borta, A., Scheltens, P., Stam, C.J., van der Flier, W.M., 2017. EEG spectral analysis as a 
putative early prognostic biomarker in nondemented, amyloid positive subjects. Neurobiol. Aging 57, $133 e 142$.

Gurevicius, K., Lipponen, A., Tanila, H., 2013. Increased cortical and thalamic excitability in freely moving APPswe/PS1dE9 mice modeling epileptic activity associated with Alzheimer's disease. Cereb. Cortex. 23, $1148 \mathrm{e} 1158$.

Habert, M.O., Bertin, H., Labit, M., Diallo, M., Marie, S., Martineau, K., Kas, A., Causse-Lemercier, V., Bakardjian, H., Epelbaum, S., Chételat, G., Houot, M., Hampel, H., Dubois, B., Mangin, J.F., INSIGHT-AD study group, 2018. Evaluation of amyloid status in a cohort of elderly individuals with memory complaints: validation of the method of quantification and determination of positivity thresholds. Ann. Nucl. Med. 32, $75 \mathrm{e} 86$.

Hall, C.B., Derby, C., LeValley, A., Katz, M.J., Verghese, J., Lipton, R.B., 2007. Education delays accelerated decline on a memory test in persons who develop dementia. Neurology 69, $1657 \mathrm{e} 1664$.

Helzner, E.P., Luchsinger, J.A., Scarmeas, N., Cosentino, S., Brickman, A.M., Glymour, M.M., Stern, Y., 2009. Contribution of vascular risk factors to the progression in Alzheimer disease. Arch. Neurol. 66, $343 e 348$.

Horváth, A., Szùcs, A., Barcs, G., Noebels, J.L., Kamondi, A., 2016. Epileptic seizures in Alzheimer disease: a review. Alzheimer Dis. Assoc. Disord. 30, 186e192.

Horvath, A., Szucs, A., Csukly, G., Sakovics, A., Stefanics, G., Kamondi, A., 2018. EEG and ERP biomarkers of Alzheimer's disease: a critical review. Front. Biosci. (Landmark Ed.) 23, 183e220.

Hughes, S.W., Crunelli, V., 2005. Thalamic mechanisms of EEG alpha rhythms and their pathological implications. Neuroscientist $11,357 e 372$.

Jack Jr., C.R., Bennett, D.A., Blennow, K., Carrillo, M.C., Dunn, B., Haeberlein, S.B., Holtzman, D.M., Jagust, W., Jessen, F., Karlawish, J., Liu, E., Molinuevo, J.L., Montine, T., Phelps, C., Rankin, K.P., Rowe, C.C., Scheltens, P., Siemers, E., Snyder, H.M., Sperling, R., Contributors, 2018. NIA-AA Research Framework: toward a biological definition of Alzheimer's disease. Alzheimers Dement. 14, $535 \mathrm{e} 562$.

Jelic, V., Johansson, S.E., Almkvist, O., Shigeta, M., Julin, P., Nordberg, A., Winblad, B., Wahlund, L.O., 2000. Quantitative electroencephalography in mild cognitive impairment: longitudinal changes and possible prediction of Alzheimer's disease. Neurobiol. Aging 21, 533e540.

Jeong, J., 2004. EEG dynamics in patients with Alzheimer's disease. Clin. Neurophysiol. 115, 1490 1505.

Jovicich, J., Babiloni, C., Ferrari, C., Marizzoni, M., Moretti, D.V., Del Percio, C., Lizio, R., Lopez, S., Galluzzi, S., Albani, D., Cavaliere, L., Minati, L., Didic, M., Fiedler, U., Forloni, G., Hensch, T., Molinuevo, J.L., Bartrés Faz, D., Nobili, F., Orlandi, D., Parnetti, L., Farotti, L., Costa, C., Payoux, P., Rossini, P.M., Marra, C., Schönknecht, P., Soricelli, A., Noce, G., Salvatore, M., Tsolaki, M., Visser, P.J., Richardson, J.C., Wiltfang, J., Bordet, R., Blin, O., Frisoniand, G.B., the PharmaCog Consortium, 2019. Two-year longitudinal monitoring of Amnestic mild cognitive impairment patients with prodromal Alzheimer's disease using topographical biomarkers derived from functional magnetic resonance imaging and electroencephalographic activity. J. Alzheimers Dis. 69, 15 e35.

Khanna, A., Pascual-Leone, A., Michel, C.M., Farzan, F., 2015. Microstates in resting-state EEG: current status and future directions. Neurosci. Biobehav Rev. 49, $105 \mathrm{e} 113$.

Klimesch, W., 1999. EEG alpha and theta oscillations reflect cognitive and memory performance: a review and analysis. Brain Res. Rev. 29, $169 \mathrm{e} 195$.

Klimesch, W., Doppelmayr, M., Schimke, H., Pachinger, T., 1996. Alpha frequency, reaction time, and the speed of processing information. J. Clin. Neurophysiol. 13, 511e518.

Kogan, E.A., Korczyn, A.D., Virchovsky, R.G., Klimovizky, S.S., Treves, T.A., Neufeld, M.Y., 2001. EEG changes during long-term treatment with donepezil in Alzheimer's disease patients. J. Neural Transm. (Vienna) 108, 1167 1173.

Kudoh, M., Takahashi, S., Yonezawa, H., 1997. [Correlation between quantitative EEG and cerebral blood flow and oxygen metabolism in patients with dementia of Alzheimer type]. Rinsho Shinkeigaku 37, 359e365. Japanese.

Lehmann, D., Ozaki, H., Pal, I., 1987. EEG alpha map series: brain micro-states by space-oriented adaptive segmentation. Electroencephalogr. Clin. Neurophysiol. 67, $271 \mathrm{e} 288$.

Liu, Y., Julkunen, V., Paajanen, T., Westman, E., Wahlund, L.O., Aitken, A., Sobow, T., Mecocci, P., Tsolaki, M., Vellas, B., Muehlboeck, S., Spenger, C., Lovestone, S., Simmons, A., Soininen, H., AddNeuroMed Consortium, 2012. Education increases reserve against Alzheimer's disease-evidence from structural MRI analysis. Neuroradiology 54, 929e938.

López-Sanz, D., Bruña, R., Garcés, P., Camara, C., Serrano, N., Rodríguez-Rojo, I.C., Delgado, M.L., Montenegro, M., López-Higes, R., Yus, M., Maestú, F., 2016. Alpha band disruption in the AD-continuum starts in the Subjective Cognitive Decline stage: a MEG study. Sci. Rep. 6, 37685.

Lorincz, M.L., Kékesi, K.A., Juhász, G., Crunelli, V., Hughes, S.W., 2009. Temporal framing of thalamic relay־mode firing by phasic inhibition during the alpha rhythm. Neuron 63, $683 \mathrm{e} 696$.

Lörincz, M.L., Crunelli, V., Hughes, S.W., 2008. Cellular dynamics of cholinergically induced alpha (8-13 Hz) rhythms in sensory thalamic nuclei in vitro. J. Neurosci. 28, $660 \mathrm{e} 671$.

Maris, E., Oostenveld, R., 2007. Nonparametric statistical testing of EEG- and MEG- data. J. Neurosci. Methods 164, 177e190.

Marizzoni, M., Ferrari, C., Macis, A., Jovicich, J., Albani, D., Babiloni, C., Cavaliere, L., Didic, M., Forloni, G., Galluzzi, S., Hoffmann, K.T., Molinuevo, J.L., Nobili, F., Parnetti, L., Payoux, P., Pizzini, F., Rossini, P.M., Salvatore, M., Schönknecht, P., Soricelli, A., Del Percio, C., Hensch, T., Hegerl, U., Tsolaki, M., Visser, P.J., Wiltfang, J., Richardson, J.C., Bordet, R., Blin, O., Frisoni, G.B., PharmaCog Consortium, 2019. Biomarker matrix to track short term disease progression in Amnestic mild cognitive impairment patients with prodromal Alzheimer's disease. J. Alzheimers Dis. 69, $49 \mathrm{e} 58$.

McKhann, G.M., Knopman, D.S., Chertkow, H., Hyman, B.T., Jack Jr., C.R., Kawas, C.H., Klunk, W.E., Koroshetz, W.J., Manly, J.J., Mayeux, R., Mohs, R.C., Morris, J.C., Rossor, M.N., Scheltens, P., Carrillo, M.C., Thies, B., Weintraub, S., Phelps, C.H., 2011. The diagnosis of dementia due to Alzheimer's disease: recommendations from the National Institute on Aging-Alzheimer's Association workgroups on diagnostic guidelines for Alzheimer's disease. Alzheimers Dement. 7, $263 e 269$.

Meng, X., D’Arcy, C., 2013. Apolipoprotein E gene, environmental risk factors, and their interactions in dementia among seniors. Int. J. Geriatr. Psychiatry 28, 1005 e1014.

Michel, C.M., Koenig, T., 2018. EEG microstates as a tool for studying the temporal dynamics of whole-brain neuronal networks: a review. Neuroimage 180 (Pt B), 577 e593.

Micotti, E., Paladini, A., Balducci, C., Tolomeo, D., Frasca, A., Marizzoni, M., Filibian, M., Caroli, A., Valbusa, G., Dix, S., O’Neill, M., Ozmen, L., Czech, C., Richardson, J.C., Frisoni, G.B., Forloni, G., 2015. Striatum and entorhinal cortex atrophy in AD mouse models: MRI comprehensive analysis. Neurobiol. Aging 36 (2), $776 \mathrm{e} 788$. 
Mortamais, M., Portet, F., Brickman, A.M., Provenzano, F.A., Muraskin, J., Akbaraly, T.N., Berr, C., Touchon, J., Bonafé, A., le Bars, E., Menjot de Champfleur, N., Maller, J.J., Meslin, C., Sabatier, R., Ritchie, K., Artero, S., 2014. Education modulates the impact of white matter lesions on the risk of mild cognitive impairment and dementia. Am. J. Geriatr. Psychiatry 22, $1336 \mathrm{e} 1345$.

Mungas, D., Gavett, B., Fletcher, E., Farias, S.T., DeCarli, C., Reed, B., 2018. Education amplifies brain atrophy effect on cognitive decline: implications for cognitive reserve. Neurobiol. Aging 68, 142e150.

Nakamura, A., Cuesta, P., Fernández, A., Arahata, Y., Iwata, K., Kuratsubo, I., Bundo, M., Hattori, H., Sakurai, T., Fukuda, K., Washimi, Y., Endo, H., Takeda, A., Diers, K., Bajo, R., Maestú, F., Ito, K., Kato, T., 2018. Electromagnetic signatures of the preclinical and prodromal stages of Alzheimer's disease. Brain 141, 1470e1485.

Nichols, T.E., Holmes, A.P., 2002. Nonparametric permutation tests for functional neuroimaging: a primer with examples. Hum. Brain Mapp. 15, $1 \mathrm{e} 25$.

Nyberg, L., Lövdén, M., Riklund, K., Lindenberger, U., Bäckman, L., 2012. Memory aging and brain maintenance. Trends Cogn. Sci. 16, $292 \mathrm{e} 305$.

Onofrj, M., Luciano, A.L., Thomas, A., Iacono, D., D’Andreamatteo, G., 2003. Mirtazapine induces REM sleep behavior disorder (RBD) in parkinsonism. Neurology 60, $113 \mathrm{e} 115$.

Oostenveld, R., Fries, P., Maris, E., Schoffelen, J.M., 2011. Fieldtrip: open source software for advanced analysis of MEG, EEG, and invasive electrophysiological data. Comput. Intell. Neurosci. 9, 156869.

Palop, J.J., Mucke, L., 2009. Epilepsy and cognitive impairments in Alzheimer disease. Arch. Neurol. 66, 435e440.

Pettigrew, C., Soldan, A., Zhu, Y., Wang, M.C., Brown, T., Miller, M., Albert, M., BIO-CARD Research Team, 2017. Cognitive reserve and cortical thickness in pre-clinical Alzheimer's disease. Brain Imaging Behav. 11, 357e367.

Pfurtscheller, G., Lopes da Silva, F.H., 1999. Event-related EEG/MEG synchronization and desynchronization: basic principles. Clin. Clin. Neurophysiol. 110, $1842 \mathrm{e} 1857$.

Ponomareva, N.V., Selesneva, N.D., Jarikov, G.A., 2003. EEG alterations in subjects at high familial risk for Alzheimer's disease. Neuropsychobiology 48, $152 \mathrm{e} 159$.

Querbes, O., Aubry, F., Pariente, J., Lotterie, J.A., Démonet, J.F., Duret, V., Puel, M., Berry, I., Fort, J.C., Celsis, P., Alzheimer's Disease Neuroimaging Initiative, 2009. Early diagnosis of Alzheimer's disease using cortical thickness: impact of cognitive reserve. Brain 132 (Pt 8), 2036e2047.

Reed, B.R., Mungas, D., Farias, S.T., Harvey, D., Beckett, L., Widaman, K., Hinton, L., DeCarli, C., 2010. Measuring cognitive reserve based on the decomposition of episodic memory variance. Brain 133 (Pt 8), $2196 \mathrm{e} 2209$.

Reeves, S., Koppel, I., Barr, H., Freeth, D., Hammick, M., 2002. Twelve tips for undertaking a systematic review. Med. Teach. 24, $358 \mathrm{e} 363$.

Rodriguez, G., Nobili, F., Rocca, G., De Carli, F., Gianelli, M.V., Rosadini, G., 1998. Quantitative electroencephalography and regional cerebral blood flow: discriminant analysis between Alzheimer's patients and healthy controls. Dement. Geriatr. Cogn. Disord. 9, 274e283.

Rodriguez, G., Nobili, F., Copello, F., Vitali, P., Gianelli, M.V., Taddei, G., Catsafados, E., Mariani, G., 1999. 99mTc-HMPAO regional cerebral blood flow and quantitative electroencephalography in Alzheimer's disease: a correlative study. J. Nucl. Med. 40, 522e529.

Rodriguez, G., Vitali, P., De Leo, C., De Carli, F., Girtler, N., Nobili, F., 2002. Quantitative EEG changes in Alzheimer patients during long-term donepezil therapy. Neuropsychobiology 46, $49 \mathrm{e} 56$.

Rodriguez, G., Vitali, P., Canfora, M., Calvini, P., Girtler, N., De Leo, C., Piccardo, A., Nobili, F., 2004. Quantitative EEG and perfusional single photon emission computed tomography correlation during long-term donepezil therapy in Alzheimer's disease. Clin. Neurophysiol. 115, 39e49.

Roe, C.M., Xiong, C., Miller, J.P., Morris, J.C., 2007. Education and Alzheimer disease without dementia: support for the cognitive reserve hypothesis. Neurology 68, $223 \mathrm{e} 228$.

Scarmeas, N., Albert, S.M., Manly, J.J., Stern, Y., 2006. Education and rates of cognitive decline in incident Alzheimer's disease. J. Neurol. Neurosurg. Psychiatry 77, $308 \mathrm{e} 316$.

Seo, S.W., Im, K., Lee, J.M., Kim, S.T., Ahn, H.J., Go, S.M., Kim, S.H., Na, D.L., 2011. Effects of demographic factors on cortical thickness in Alzheimer's disease. Neurobiol. Aging 32, 200e209.

Stern, Y., 2012. Cognitive reserve in ageing and Alzheimer's disease. Lancet Neurol. 11, $1006 \mathrm{e} 1012$.

Stern, Y., Albert, S.M., Tang, M.X., Tsai, W.Y., 1999. Rate of memory decline in AD is related to education and occupation: cognitive reserve? Neurology 53, $1942 \mathrm{e} 1947$.

Stern, Y., Arenaza-Urquijo, E.M., Bartrés-Faz, D., Belleville, S., Cantilon, M., Chetelat, G., Ewers, M., Franzmeier, N., Kempermann, G., Kremen, W.S., Okonkwo, O., Scarmeas, N., Soldan, A., Udeh-Momoh, C., Valenzuela, M., Vemuri, P., Vuoksimaa, E., Reserve, Resilience and Protective Factors PIA Empirical Definitions and Conceptual Frameworks Workgroup, 2018. White-paper: defining and investigating cognitive reserve, brain reserve, and brain maintenance. Alzheimers Dement. S1552-5260, 33491e33495.

Szelies, B., Mielke, R., Kessler, J., Heiss, W.D., 1999. EEG power changes are related with regional cerebral glucose metbolism in vascular dementia. Clin. Neurophysiol. 110, $615 \mathrm{e} 620$.

Teipel, S., Bakardjian, H., Gonzalez-Escamilla, G., Cavedo, E., Weschke, S., Dyrba, M., Grothe, M.J., Potier, M.C., Habert, M.O., Dubois, B., Hampel, H., INSIGHT-preAD Study Group, 2017a. No association of cortical amyloid load and EEG connectivity in older people with subjective memory complaints. Neuroimage Clin. 17, 435e443.

Teipel, S.J., Cavedo, E., Weschke, S., Grothe, M.J., Rojkova, K., Fontaine, G., Dauphinot, L., Gonzalez-Escamilla, G., Potier, M.C., Bertin, H., Habert, M.O., Dubois, B., Hampel, H., INSIGHT-preAD Study Group, 2017b. Cortical amyloid accumulation is associated with alterations of structural integrity in older people with subjective memory complaints. Neurobiol. Aging $57,143 \mathrm{e} 152$

Thomas, B.A., Erlandsson, K., Modat, M., Thurfjell, L., Vandenberghe, R., Ourselin, S., Hutton, B.F., 2011. The importance of appropriate partial volume correction for PET quantification in Alzheimer's disease. Eur. J. Nucl. Med. Mol. Imaging 38, $1104 \mathrm{e} 1119$

Valladares-Neto, D.C., Buchsbaum, M.S., Evans, W.J., Nguyen, D., Nguyen, P., Siegel, B.V., Stanley, J., Starr, A., Guich, S., Rice, D., 1995. EEG delta,positron emission tomography, and memory deficit Alzheimer's disease. Neuropsychobiology 31 , $173 \mathrm{e} 181$. 
Vaqué-Alcázar, L., Sala-Llonch, R., Valls-Pedret, C., Vidal-Piñeiro, D., Fernández-Cabello, S., Bargalló, N., Ros, E., Bartrés-Faz, D., 2017. Differential age-related gray and white matter impact mediates educational influence on elders' cognition. Brain Imaging Behav. 11, 318e332.

Vossel, K.A., Ranasinghe, K.G., Beagle, A.J., Mizuiri, D., Honma, S.M., Dowling, A.F., Darwish, S.M., Van Berlo, V., Barnes, D.E., Mantle, M., Karydas, A.M., Coppola, G., Roberson, E.D., Miller, B.L., Garcia, P.A., Kirsch, H.E., Mucke, L., Nagarajan, S.S., 2016. Incidence and impact of subclinical epileptiform activity in Alzheimer's disease. Ann. Neurol. 80, 858e870.

Wada, M., Noda, Y., Shinagawa, S., Chung, J.K., Sawada, K., Ogyu, K., Tarumi, R., Tsugawa, S., Miyazaki, T., Yamagata, B., GraffGuerrero, A., Mimura, M., Nakajima, S., Alzheimer's Disease Neuroimaging Initiative, 2018. Effect of education on Alzheimer's disease-related neuroimaging biomarkers in healthy controls, and participants with mild cognitive impairment and Alzheimer's disease: a cross-sectional study. J. Alzheimers Dis. 63, 861e869.

Wan, L., Huang, H., Schwab, N., Tanner, J., Rajan, A., Lam, N.B., Zaborszky, L., Li, C.R., Price, C.C., Ding, M., 2019. From eyesclosed to eyes-open: role of cholinergic projections in EC-to-EO alpha reactivity revealed by combining EEG and MRI. Hum. Brain Mapp. 40, 566e577.

Weiner, P., Waizman, J., Weiner, M., Rabner, M., Magadle, R., Zamir, D., 1998. Influence of excessive weight loss after gastroplasty for morbid obesity on respiratory muscle performance. Thorax 53, 39e42.

Wilson, R.S., Yu, L., Lamar, M., Schneider, J.A., Boyle, P.A., Bennett, D.A., 2019. Education and cognitive reserve in old age. Neurology 92, e1041ee1050.

Wilson, R.S., Bennett, D.A., Gilley, D.W., Beckett, L.A., Barnes, L.L., Evans, D.A., 2000. Premorbid reading activity and patterns of cognitive decline in Alzheimer disease. Arch. Neurol. 57, 1718e1723.

Wilson, R.S., Li, Y., Aggarwal, N.T., Barnes, L.L., McCann, J.J., Gilley, D.W., Evans, D.A., 2004. Education and the course of cognitive decline in Alzheimer disease. Neurology 63, 1198e1202.

Zahodne, L.B., Manly, J.J., Brickman, A.M., Siedlecki, K.L., Decarli, C., Stern, Y., 2013. Quantifying cognitive reserve in older adults by decomposing episodic memory variance: replication and extension. J. Int. Neuropsychol. Soc. 19, 854 e862.

Zahodne, L.B., Manly, J.J., Brickman, A.M., Narkhede, A., Griffith, E.Y., Guzman, V.A., Schupf, N., Stern, Y., 2015. Is residual memory variance a valid method for quantifying cognitive reserve? A longitudinal application. Neuropsychologia 77, $260 \mathrm{e} 266$.

Zahodne, L.B., Mayeda, E.R., Hohman, T.J., Fletcher, E., Racine, A.M., Gavett, B., Manly, J.J., Schupf, N., Mayeux, R., Brickman, A.M., Mungas, D., 2019. The role of education in a vascular pathway to episodic memory: brain maintenance or cognitive reserve? Neurobiol. Aging 84, 109e118. 